\title{
Prompt $J / \psi$-pair production at the LHC: impact of loop-induced contributions and of the colour-octet mechanism
}

\author{
Jean-Philippe Lansberg ${ }^{1}$, Hua-Sheng Shao ${ }^{2, a}$, Nodoka Yamanaka ${ }^{1,3}$, Yu-Jie Zhang ${ }^{4,5}$ \\ ${ }^{1}$ IPNO, CNRS-IN2P3, Univ. Paris-Sud, Université Paris-Saclay, 91406 Orsay Cedex, France \\ ${ }^{2}$ Laboratoire de Physique Théorique et Hautes Energies (LPTHE), UMR 7589, Sorbonne Université et CNRS, 4 place Jussieu, 75252 Paris Cedex \\ 05, France \\ ${ }^{3}$ Yukawa Institute for Theoretical Physics, Kyoto University, Kitashirakawa-Oiwake, Kyoto 606-8502, Japan \\ ${ }^{4}$ School of Physics and Nuclear Energy Engineering, Beihang University, Beijing 100083, China \\ ${ }^{5}$ Center for High Energy Physics, Peking University, Beijing 100871, China
}

Received: 8 August 2019 / Accepted: 29 November 2019 / Published online: 13 December 2019

(C) The Author(s) 2019

\begin{abstract}
Prompt double- $J / \psi$ production at high-energy hadron colliders can be considered as a golden channel to probe double parton scatterings (DPS) - in particular to study gluon-gluon correlations inside the proton-and, at the same time, to measure the distribution of linearly-polarised gluons inside the proton. Such studies, however, require a good control of both single parton scatterings (SPS) and DPS in the respective regions where they are carried out. In this context, we have critically examined two mechanisms of SPS that may be kinematically enhanced where DPS are thought to be dominant, even though they are either at higher orders in the strong-coupling or velocity expansion. First, we have considered a gauge-invariant and infrared-safe subset of the loopinduced contribution via colour-singlet (CS) transitions. We have found it to become the leading CS SPS contributions at large rapidity separation, yet too small to account for the data without invoking the presence of DPS yields. Second, we have surveyed the possible colour-octet (CO) contributions using both old and up-to-date non-perturbative longdistance matrix elements (LDMEs). We have found that the pure $\mathrm{CO}$ yields crucially depend on the LDMEs. Among all the LDMEs we used, only two result into a visible modification of the NRQCD $(\mathrm{CS}+\mathrm{CO})$ yield, but only in two kinematical distributions measured by ATLAS, those of the rapidity separation and of the pair invariant mass. These modifications, however, do not impact the control region used for their DPS study.
\end{abstract}

\section{Introduction}

The role of multiple parton interactions in proton-proton collisions is believed to become increasingly important when one explores the energy frontier in particle physics. As such, the relevance in LHC observables of two simultaneous hard scatterings, usually referred to as double parton scatterings (DPS), has attracted much attention in the last decade with significant theory advances related to perturbative QCD [115]. Since DPS are higher-twist effects in total cross sections compared to the conventional single parton scatterings (SPS), quantitative studies of DPS remain challenging though not impossible both on the theoretical and experimental sides. These are particularly interesting since they provide us with means to study parton correlations inside the proton (see e.g. $[10,16,17])$.

Among the possible hard probes of DPS at high-energy hadron colliders, the associated production of quarkonia (see [18] for an exhaustive review) provides unique opportunities to measure DPS in gluon-induced reactions thus to study gluon-gluon correlations in the proton. Numerous measurements of quarkonium associated processes have been performed at the Tevatron and the LHC. They can mainly be categorised as di-quarkonium production $(J / \psi+J / \psi[19$ 23], $J / \psi+\Upsilon$ [24], $\Upsilon+\Upsilon$ [25]), associated production with a vector boson $\left(J / \psi+W^{ \pm}[26], J / \psi+Z\right.$ [27]) or with another heavy quark ( $J / \psi+$ open charm [28], $\Upsilon+$ open charm [29]). All these measurements cover different kinematical regions with different momentum transfers in the hard scattering. Their theoretical analysis is highly non-trivial, which has

ae-mail: hshao@lpthe.jussieu.fr 
triggered many theoretical studies in the recent years [30$41,41-51]$. Very recently, the first calculation of triple- $J / \psi$ production showed that it can help us probe both DPS and triple parton scatterings (TPS) [52].

In this context, we focus in this paper on the di- $J / \psi$ case with the aim to improve the existing perturbative QCD calculations for the SPS. To do so, we consider higher-order corrections in both the strong-coupling constant, $\alpha_{S}$, and the heavy-quark velocity, $v$. First, we study the impact of a gauge-invariant and infrared-safe subset of loop-induced (LI) contributions. Our analysis follows the lines of a similar study for $J / \psi+\Upsilon$ production [42]. Such contributions appear at next-to-next-to-leading order (NNLO) in $\alpha_{S}$ but could be enhanced at large rapidity differences and high invariant masses of the $J / \psi$ pair because of the presence of topologies with double $t$-channel gluon exchanges between both charm-anticharm-quark lines. Second, we perform a comprehensive survey of the impact of the colour-octet (CO) contributions in three kinematical domains covered by the existing LHC measurements [21-23] considering the various existing fits of the non-perturbative $\mathrm{CO}$ long-distance matrix elements (LDMEs).

In order to disentangle DPS from SPS in observables where two particles are observed, one usually relies on the analysis of specific kinematical dependences which are believed to be drastically different in both samples. Common choices of variables are the azimuthal and the rapidity separations between both observed particles, $\Delta \phi$ and $\Delta y$. The DPS contribution, coming from two a priori independent parton scatterings, is expected to be flatter than the SPS one in both distributions.

For double- $J / \psi$ studies, the analysis of the $\Delta y(J / \psi, J / \psi)$ distributions should be preferred compared to that of $\Delta \phi(\mathrm{J} /$ $\psi, J / \psi)$ since the $\Delta y(J / \psi, J / \psi)$ distribution of the SPS yield is much less affected by possible non-perturbative intrinsic $k_{T}$ of the colliding gluons [33] than the $\Delta \phi(J / \psi, J /$ $\psi$ ) one, which can become as flat as the DPS ones in some cases. In general, one expects the DPS fraction to be the largest at large $|\Delta y(J / \psi, J / \psi)|$. A precise determination of the DPS yield therefore requires good knowledge of the SPS in this region. Both the LI and CO topologies with $t$ channel gluon exchanges could result into a flat $\frac{d \sigma}{d \Delta y}$ like in the $J / \psi+\Upsilon$ case [42].

Assuming $\alpha_{S} \sim v^{2}$, the colour-singlet (CS) LI contribution should be of the same magnitude as the leading order (LO) CO contribution (yet both smaller than the bulk of the $\mathrm{CS}$ yield in the absence of the possible kinematical enhancement which we are after here). According to the NRQCD velocity scaling rules [53], the former one is indeed $\mathcal{O}\left(\alpha_{S}^{6} v^{3}\right)$ while the latter one is $\mathcal{O}\left(\alpha_{S}^{4} v^{7}\right)$. This justifies why we consider both of them in this study.

This article is organised as follows. In Sect. 2, we first quickly review the existing LHC measurements used in our
Table 1 Summary of the kinematical variables

\begin{tabular}{ll}
\hline$P_{T}(J / \psi+J / \psi)$ & $y(J / \psi+J / \psi)$ \\
$\begin{array}{l}\text { Transverse momentum of } \\
\text { the pair }\end{array}$ & Rapidity of the pair \\
$P_{T}(J / \psi)$ & $y(J / \psi)$ \\
Transverse momentum of a & Rapidity of a randomly cho- \\
randomly chosen $J / \psi$ & $\operatorname{sen} J / \psi$ \\
$\Delta \phi(J / \psi, J / \psi)$ & $\Delta y(J / \psi, J / \psi)$ \\
Azimuthal angle difference & Rapidity separation \\
in the transverse plane & \\
$M(J / \psi+J / \psi)$ & $A_{T}(J / \psi, J / \psi)$ \\
Invariant mass of the pair & Transverse-momentum \\
& asymmetry
\end{tabular}

comparisons. ${ }^{1}$ Then, we discuss our theory framework in Sect. 3. Section 4 gathers our discussion of the impact of the inclusion LI CS contribution and Sect. 5 comprises a comprehensive analysis of complete LO CO contribution. In Appendix A, we additionally show results with a hybrid CO LDME set. Appendix B collects additional plots relevant for further theory-data comparisons.

\section{LHC measurements and kinematical variables}

\subsection{Kinematical variables}

We start by introducing the kinematical variables relevant for di-quarkonium production. On the experimental side, the second LHCb analysis [23] bears on the largest set of the kinematical variables whose distribution is used for comparisons between the experimental measurements and the theoretical calculations. Since some of these variables may not be very common, we summarise the description of their names or labels in Table 1. In particular, the transverse-momentum asymmetry is defined as

$A_{T}(J / \psi, J / \psi) \equiv\left|\frac{P_{T}\left(J / \psi_{1}\right)-P_{T}\left(J / \psi_{2}\right)}{P_{T}\left(J / \psi_{1}\right)+P_{T}\left(J / \psi_{2}\right)}\right|$,

where $J / \psi_{1}$ and $J / \psi_{2}$ are, respectively, denoted as the first and second hardest $J / \psi$ with ordering in the transverse momentum.

\subsection{Available data sets}

Four LHC studies of double prompt $J / \psi$ production have so far been performed [19,21-23]. LHCb performed two measurements in the same kinematical region, one at $\sqrt{s}=7 \mathrm{TeV}$

\footnotetext{
${ }^{1}$ We do not consider the D0 measurement [20] at the Tevatron since no corrected distribution was released which could be used for a direct data-theory comparison.
} 
Table 2 Summary of kinematical cuts of the double- $J / \psi$ measurements by the LHC experiments which we will consider here

\begin{tabular}{lll}
\hline Experiment & $\sqrt{s}(\mathrm{TeV})$ & Kinematical cuts \\
\hline CMS [21] & 7 & $P_{T}(J / \psi)>6.5 \mathrm{GeV}$ when $|y(J / \psi)|<1.2 ;$ \\
& & $P_{T}(J / \psi)>6.5-\frac{200}{23}(|y(J / \psi)|-1.2) \mathrm{GeV}$ when $1.2<|y(J / \psi)|<1.43 ;$ \\
& $P_{T}(J / \psi)>4.5 \mathrm{GeV}$ when $1.43<|y(J / \psi)|<2.2$ \\
& $P_{T}(\mu)>2.5 \mathrm{GeV},|\eta(\mu)|<2.3 ;$ \\
& One $J / \psi$ has two muons with $P_{T}(\mu)>4 \mathrm{GeV} ;$ \\
& $P_{T}(J / \psi)>8.5 \mathrm{GeV},|y(J / \psi)|<2.1$ \\
& & $P_{T}(J / \psi)<14 \mathrm{GeV}, 2.0<y(J / \psi)<4.5$
\end{tabular}

and another at $\sqrt{s}=13 \mathrm{TeV}$; we will focus on the latter which is more precise [23]. The various kinematical cuts used in the ATLAS, CMS and LHCb analyses are summarised in Table 2 along with the corresponding centre-of-mass energy $\sqrt{s}$. It is useful to note that due to the different trigger and acceptance constraints on the ATLAS, CMS and LHCb data taking, the 3 samples cover complementary domains in $P_{T}$ and $y$. In particular, ATLAS [22] imposes the largest $P_{T}(J / \psi)$ cut (as large as $8.5 \mathrm{GeV}$ ), while $\mathrm{LHCb}$ [23] does not impose any lower $P_{T}$ cut on the observed $J / \psi$. As such, LHCb events are mostly located at low $P_{T}(J / \psi)$. CMS [21] imposes varying cuts from $P_{T}(J / \psi)>4.5 \mathrm{GeV}$ to $P_{T}(J / \psi)>$ $6.5 \mathrm{GeV}$ depending on the rapidity. Moreover, LHCb can only detect forward particles whereas ATLAS/CMS have a generally larger rapidity coverage but in the centralrapidity region. In Sect. 5, we will discuss how these kinematical coverages can be relevant to determine the proper CO LDMEs.

\section{Theory framework}

In this section, we briefly address some specificities of our theoretical computations, which, however, remain very standard.

\subsection{Intrinsic initial- $k_{T}$ smearing}

An important effect for an accurate description of double$J / \psi$ hadroproduction is known to be the smearing of the kinematics arising from the intrinsic $k_{T}$ of the gluons [54]. It is in principle a non-perturbative effect which cannot properly be accounted for by the collinear factorisation. In fact, double- $J / \psi$ production can provide new insights in the transverse dynamics of the gluons as it was shown [48] using the transverse-momentum dependent (TMD) factorisation. Clearly, a collinear computation is not meant to encapsulate such effects. As a makeshift, we simply rely on an empirical procedure to deal with them which we believe to be sufficient for our phenomenological purpose. In particular, the whole $k_{T}$ smearing is assumed to be factorised out by $\frac{d \sigma}{d \Phi_{\left\langle k_{T}\right\rangle}}=\int_{0}^{+\infty} d k_{T}^{2} \frac{\pi}{8\left\langle k_{T}\right\rangle^{2}} e^{-\frac{\pi}{8} \frac{k_{T}^{2}}{\left\langle k_{T}\right\rangle^{2}}} \frac{d \sigma}{d \Phi}$,

where the phase-space mapping $\Phi \rightarrow \Phi_{\left\langle k_{T}\right\rangle}$ is determined by boosting the whole event according to the generated transverse-momentum imbalance $\left|\overrightarrow{k_{T}}\right|=k_{T}$ with a uniform distribution of the azimuthal angle in the transverse plane. Other forms are of course possible. In the present study, we assume $\left\langle k_{T}\right\rangle$ to be the same for all three experimental coverages and fix its value to be $3.0 \mathrm{GeV}$. The distributions with other $\left\langle k_{T}\right\rangle$ values are also not shown but can easily be obtained with the help of HELAC-ONIA $[55,56]$. In fact, the $\mathrm{NLO}^{\star}$ distributions with $\left\langle k_{T}\right\rangle=0.5 \mathrm{GeV}$ and $2.0 \mathrm{GeV}$ can be found in a theory-data comparison made by $\mathrm{LHCb}$ [23]. The $k_{T}$-smearing effect is only visible for the $P_{T}(J / \psi+J / \psi)$, $\Delta \phi(J / \psi, J / \psi)$ and $A_{T}(J / \psi, J / \psi)$ distributions.

\subsection{Parameters entering our calculations}

We now quickly describe our set-up for the present calculations before discussing the numerical results. We have fixed the charm-quark mass to be $1.5 \mathrm{GeV}$ and only the light $u, d$, $s$ (anti)quarks and the gluons are allowed in the initial states. In order to be compatible with our previous $\mathrm{NLO}^{\star}$ calculations, we have used the NLO parton-distribution functions (PDFs) CTEQ6M [57] for the calculations in the ATLAS and CMS acceptances and NNPDF3.0 [58] for those in the $\mathrm{LHCb}$ acceptance. We have explicitly computed that the PDF uncertainty from NNPDF3.0 is less than 20\%, whereas the difference between CTEQ6M and NNPDF3.0 PDF sets is below $15 \%$ at $13 \mathrm{TeV}$ in the $\mathrm{LHCb}$ acceptance, which lies within the estimated NNPDF PDF uncertainty. Since the main PDF uncertainty is from the least constrained low-x gluon density, we anticipate that the PDF uncertainty should be less than $15 \%$ in the ATLAS and CMS acceptance which correspond to larger $\mathrm{x}$ and larger scales. Such an uncertainty is thus in general small compared to the (dominant) scale uncertainty which we discuss below. The missing higher-order terms in $\alpha_{S}$ are estimated in the usual way by independently varying the factorisation and renormalisation scales as $\left(\mu_{F}, \mu_{R}\right)=\left(\zeta_{1} \mu_{0}, \zeta_{2} \mu_{0}\right)$, 
with $\zeta_{1,2}=\frac{1}{2}, 1,2$, where the central scale $\mu_{0}$ is chosen to be $\mu_{0}=\sqrt{\left(P_{T}(J / \psi)\right)^{2}+\left(4 m_{c}\right)^{2}}$, like in Refs. [35, 37]. The CS LDME is estimated via $\left\langle\mathcal{O}^{H_{Q} \bar{Q}}\left({ }^{3} S_{1}^{[1]}\right)\right\rangle=$

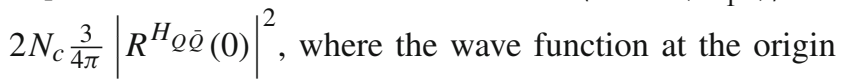
$R^{H_{Q} \bar{Q}(0)}$ can be determined by solving the Schrödinger equation with a given QCD potential. We will use the numerical values $\left|R^{J / \psi}(0)\right|^{2}=0.8 \mathrm{GeV}^{3}$ and $\left|R^{\psi(2 S)}(0)\right|^{2}=$ $0.5 \mathrm{GeV}^{3}$ derived in Ref. [59] using the QCD-motivated Buchmüller-Tye potential [60]. For the CS SPS yield, the feed-down contribution from the $\psi(2 S)$ decays is as large as the direct double $J / \psi$ production. In practice, we take it to be equal to 2 . It is thus mandatory to take it into account.

\section{Colour-singlet contributions: partial loop-induced corrections}

In principle, considering the square of a one-loop amplitude by itself should give divergent results from both the infrared and the ultraviolet regions. Such one-loop amplitudes squared are part of the NNLO contributions, at $\mathcal{O}\left(\alpha_{S}^{6}\right)$ in the case of double $J / \psi$ hadroproduction. The cancellation of the aforementioned infrared divergences would be achieved as usual by considering two-loop, one-loop singlereal-emission and double-real-emission amplitudes. Such a computation is obviously beyond the scope of this study-it is not even available for single $J / \psi$.

However, a subset of such one-loop diagrams, restricted to the sole topologies with two separate charm-quark lines forming each a $J / \psi$, happens to be free of any divergence and is, in addition, gauge invariant. Correspondingly, the possible double-real emissions which could develop infrared divergences do not contribute when one of the external gluon becomes soft. This is akin to the absence of any infrared divergences at $P_{T} \rightarrow 0$ for $g g \rightarrow J / \psi g$. Such a subset is in fact that of the LI contribution to $p p \rightarrow J / \psi+\Upsilon$ considered in Ref. [42]

The square of the amplitude from these one-loop diagrams is what we here refer to as the (partial) LI corrections. Their computation is included in the HELAC- ONIA code $[55,56]$ and is thus available to everybody. In fact, another gaugeinvariant $\mathcal{O}\left(\alpha_{S}^{6}\right)$ part, namely from $p p \rightarrow J / \psi+J / \psi+c \bar{c}$, is known [37]. It turns out to be small and can safely be ignored for our purposes. However, we wish to point out that the process $p p \rightarrow J / \psi+J / \psi+c \bar{c}$ has its own interest as it can be a potential probe of the TPS at the LHC.

Let us add that we do not expect any specific kinematical enhancement of other NNLO topologies, in particular that of the double-real-gluon emission in view of the results of $p p \rightarrow J / \psi+\Upsilon[42]$. This is partly explained by the
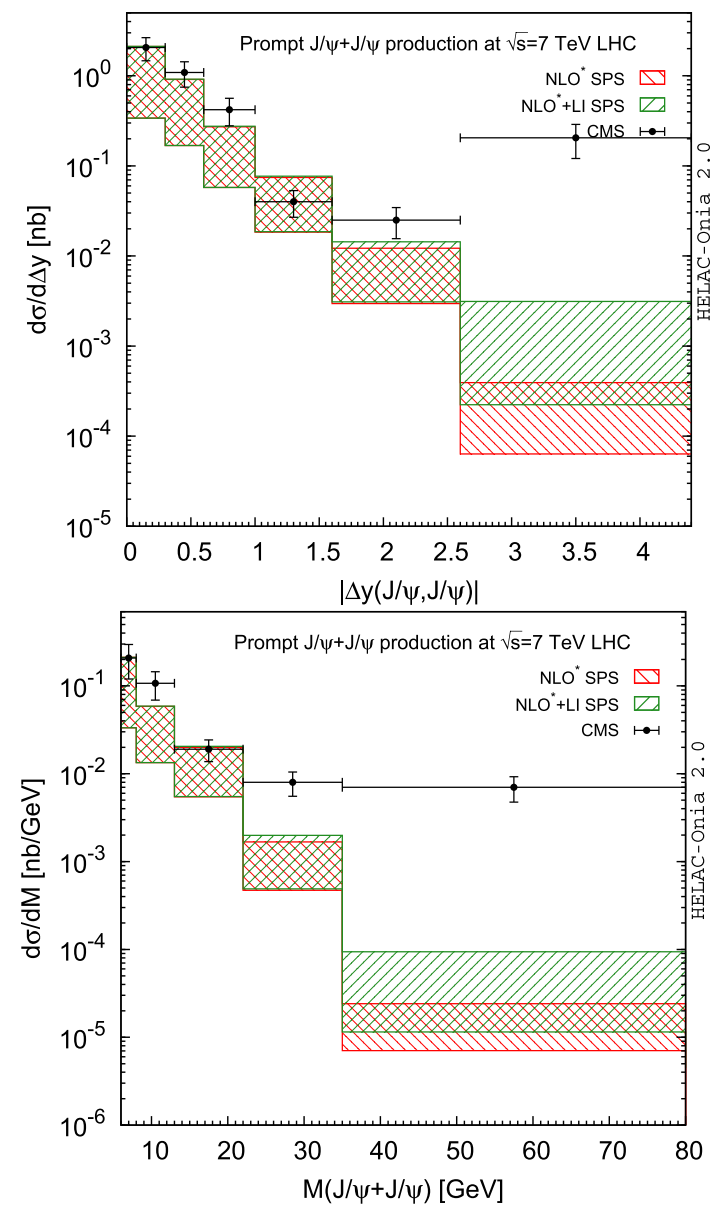

Fig. 1 Rapidity gap $|\Delta y(J / \psi, J / \psi)|$ (left) and invariant mass $M(J / \psi+J / \psi)$ (right) distributions for di- $J / \psi$ production in CSM via SPS within CMS $\sqrt{s}=7 \mathrm{TeV}$ acceptance [21]

vanishing of these contributions when one gluon becomes soft, precisely where one can minimise the off-shellness of the other particles involved in the scattering and thus where these contributions could have been the largest.

The differential cross sections in the absolute rapidity difference between the $J / \psi$ pair $|\Delta y(J / \psi, J / \psi)|$ are shown in the left panels of Figs. 1, 2 and 3 and are compared to the CMS, ATLAS and LHCb data. The NLO^ CS calculations are displayed by the red hatched bands in the figures. The partial LI contributions are represented by the green bands. As expected, the (partial) LI is significant at large $|\Delta y(J / \psi, J / \psi)|$ region but negligible at small and intermediate $|\Delta y|$. An order of magnitude enhancement to the CS cross section is expected when $|\Delta y(J / \psi, J / \psi)| \geq 3.0$. Nonetheless, despite the very large theoretical uncertainties from the scale variations, a discrepancy between the CS SPS and the experimental data is clearly visible at large $|\Delta y(J / \psi, J / \psi)|$, and that is exactly where the DPS is expected to be important. 

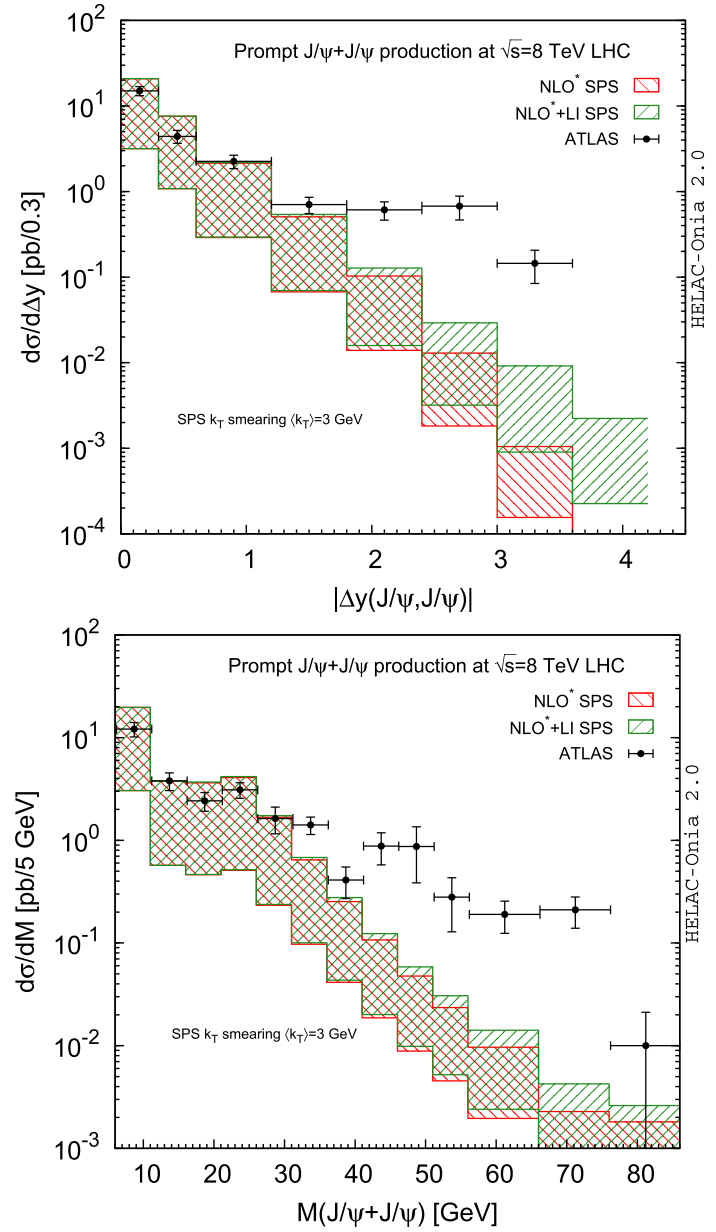

Fig. 2 Rapidity gap $|\Delta y(J / \psi, J / \psi)|$ (left) and invariant mass $M(J / \psi+J / \psi)$ (right) distributions for di- $J / \psi$ production in CSM via SPS within ATLAS $\sqrt{s}=8 \mathrm{TeV}$ acceptance [22]

The invariant mass of the meson pair is also closely related to the rapidity gap $|\Delta y(J / \psi, J / \psi)|$ (see the discussion in Ref. [37]). Large $M(J / \psi+J / \psi)$ bins are usually populated by large $|\Delta y(J / \psi, J / \psi)|$ events. Similar enhancements from the LI contributions can be seen in the tail of the invariant-mass distributions of Figs. 1 and 2. The measurements by CMS and ATLAS are consistent with the SPS CS alone at low invariant masses and depart from the SPS CS bands $\left(\mathrm{NLO}^{\star}\right.$ and $\left.\mathrm{NLO}^{\star}+\mathrm{LI}\right)$ at large $M(J / \psi+J / \psi)$ values. In contrast, for the LHCb acceptance, the LI part is negligible compared to the $\mathrm{NLO}^{\star}$ contributions due to the limited $M(J / \psi+J / \psi)$ range, below $14 \mathrm{GeV}$.

We have collected additional data-theory-comparison plots between the SPS CS yields and the LHC measurements for other observables in Appendix B.1. The data are compatible with the CS theoretical predictions but the LI contributions are found to be negligible for all the other distributions.


Fig. 3 Rapidity gap $|\Delta y(J / \psi, J / \psi)|$ (left) and invariant mass $M(J / \psi+J / \psi)$ (right) distributions for di- $J / \psi$ production in CSM via SPS within LHCb $\sqrt{s}=13 \mathrm{TeV}$ acceptance [23]

\section{Comprehensive assessment of the colour-octet contributions}

The whole LO CO contributions to di- $\psi$ hadroproduction at the LHC up to $\mathcal{O}\left(v^{7}\right)$ in NRQCD have recently been computed by He and Kniehl [40]. Their study, however, bears on a single CO LDME set from an out-of-date LO single $J / \psi$ hadroproduction fit [61] which was made with the early Tevatron data. Yet, their calculation seems to indicate that the $\mathrm{CO}$ contributions might be relevant at large $|\Delta y(J / \psi, J / \psi)|$ and large $M(J / \psi+J / \psi)$ due to similar $t$-channel gluon exchange diagrams than for the CS LI contributions. The aforementioned remaining discrepancy between this full SPS LO NRQCD calculation and the CMS data at large $|\Delta y(J / \psi, J / \psi)|$ was then attributed to unknown missing higher-order QCD corrections to the $\mathrm{CO}$ contributions.

We, however, note that we do not anticipate any such socalled "giant" $K$ factors in this region. Currently, no com- 
plete NLO CO calculation exists. Since it is important to deal with a complete set of CO channels in order to guarantee the large cancellation between $S$-wave and $P$-wave contributions involved in the NLO LDME fits of hadroproduction data, we consider that to rely on a LO_-but complete-perturbative calculation and then to estimate the size of the missing higherorder corrections via the scale uncertainty is probably the most reasonable procedure to adopt.

An alternative approach to investigating the presence of possible "giant" $K$ factors from new fragmentation topologies - if some are indeed relevant-without performing a full computation is that recently proposed by one of us in Ref. [62]. It has been proved useful for the single $J / \psi$ hadroproduction case. The method is in principle general and applicable for the double $J / \psi$ hadroproduction as well, although a new infrared divergence in double $P$-wave channels emerges [63]. We leave it for future studies since it may not apply to the whole phase space, which we wish to consider here. Finally, we note that a similar enhancement from $t$-channel gluon exchange was expected for di- $\chi_{c}$ production but its feed down was also found to be insignificant in the di- $\psi$ yield [49].

\subsection{Status and issues with the colour-octet transitions}

Although the possibility for $\mathrm{CO}$ transitions is a robust prediction from NRQCD, their actual impact in the phenomenology has been the subject of debates for decades. The most glaring observations for the necessity of their presence are twofold. First, CO provide a natural solution for the infrared divergence issue in $P$-wave production. Second, the LO $v^{2}$ NRQCD calculation involving only CS transitions still underestimates-even after including NLO QCD corrections - the yields of single $J / \psi$ and $\psi(2 S)$ hadroproduction at large $P_{T}$ at the Tevatron and the LHC.

However, NRQCD computations even including CO contributions are unable to coherently describe-i.e. with the same CO LDMEs - the world data for $p p, e p, \gamma p, \gamma \gamma$ and $e^{+} e^{-}$collisions. For a recent review, we guide the reader to Ref. [18].

The CO LDMEs are predicted to be universal nonperturbative objects by NRQCD, which should yield predictions compatible with all the data. The current status of their extractions is very confusing as their numerical values and their uncertainties are very disparate. The results of the fits of different groups disagree with each others. As long as the situation is not clarified, we believe that it is necessary to comprehensively consider these analyses instead of drawing conclusions based on a single CO LDME set as is often done in the analysis of associated production of quarkonium (see Ref. [18] for some examples).

As such, we will use different LDME sets of which we briefly review the status and the possible limitations. As we said above, the available CO LDMEs for prompt $J / \psi$ production are extracted from fits. According to the QCD accuracy of the short-distance coefficients (SDCs), we will categorise them in the four groups shown in Table 3. Namely,

1. three fits are based on LO SDCs $[61,64,65]$,

2. four fits based on NLO SDCs [67-70],

3. one fit based on a low- $P_{T}$ leading-logarithm (LL) resummed SDC [66],

4. one fit using a SDC using leading-power (LP) fragmentation matched to NLO SDC [71].

All of them have shortcomings and/or limitations. We enumerate them below:

1. First of all, we wish to emphasise that the LO fits are out-of-date and should be viewed as a pure tuning of the normalisation of the single $J / \psi$ data. Since all of the LO fits are mainly performed with the help of intermediate and large $P_{T}$ hadroproduction data, where the "giant" $K$ factors from NLO QCD corrections emerge, it is very hard to imagine that these values will give correct predictions for independent observables, like the double- $J / \psi$ hadroproduction in our case, for which $K$ factors would be different. We will therefore use them here for a pure illustrative purpose.

2. The LL fit in Ref. [66] concentrates on the $P_{T}(J / \psi)<$ $m_{c}$ region. The authors performed a small- $P_{T}$ resummation but without considering the contribution from the CS channel, which is, however, known to saturate the data in this region [72,73]. The values of these LDMEs have never been used for the single $J / \psi$ production at intermediate and large $P_{T}$ regions. They are included in our discussion like the LO fits in order to be exhaustive.

3. The NLO fit in Ref. [67] used the world data before 2011 without subtracting the feed-down contributions. The fit seems to yield good agreement with the $P_{T}(J / \psi)<30$ $\mathrm{GeV} J / \psi$ yields data at different colliders but for $\gamma \gamma$ and $e^{+} e^{-}$collisions. However, it overshoots the $P_{T}>30$ $\mathrm{GeV}$ yields and fails to reproduce the polarisations of $J / \psi$, the energy-fraction distribution of the $J / \psi$ in jets [74] and the yields of $\eta_{c}$ (by using heavy-quark spin symmetry). In addition, the SPS $P_{T}$-differential cross section of $J / \psi+\gamma$ [75] turns out to be negative at NLO with these values of CO LDMEs.

4. The NLO fit by Gong et al. [68] focus on the $P_{T}(J / \psi)>$ $7 \mathrm{GeV}$ data. The feed-down contributions are subtracted. This LDME set is, however, not compatible with the yields (e.g. $p p, \gamma p$ and $e^{+} e^{-}$) when $P_{T}(J / \psi)<7 \mathrm{GeV}$, the polarisation of forward $J / \psi[76]$ and the $\eta_{c}$ production. In addition, it yields - unphysical-negative cross sections in $p p \rightarrow J / \psi+\gamma$. In principle, this set is only applicable to $J / \psi$ production with $P_{T}(J / \psi)>7 \mathrm{GeV}$, 
Table 3 Summary of LDMEs we used from various fits [Set 1: Sharma et al. [64]; Set 2: Braaten et al. [61]; Set 3: Krämer [65]; Set 4: Sun et al. [66]; Set 5: Butenschön et al. [67]; Set 6 : Gong et al. [68]; Set 7: Shao et al. [69]: Set 8: Han et al. [70]: Set 9: Bodwin et al. [71]]

\begin{tabular}{|c|c|c|c|c|c|}
\hline & \multicolumn{4}{|l|}{ LO fits } & \multirow{2}{*}{$\frac{\text { LL fit }}{\text { Set } 4}$} \\
\hline & Set 1 & \multicolumn{2}{|c|}{ Set 2} & Set 3 & \\
\hline$\left\langle\mathcal{O}^{J / \psi}\left({ }^{3} S_{1}^{[1]}\right)\right\rangle\left(\mathrm{GeV}^{3}\right)$ & \multicolumn{2}{|l|}{$1.2 \mathrm{GeV}^{3}$} & 1.4 & 1.16 & 1.16 \\
\hline$\left\langle\mathcal{O}^{J / \psi}\left({ }^{3} S_{1}^{[8]}\right)\right\rangle\left(\mathrm{GeV}^{3}\right)$ & \multicolumn{2}{|l|}{$1.3 \times 10^{-3}$} & $3.9 \times 10^{-3}$ & $1.2 \times 10^{-2}$ & $-9.3 \times 10^{-3}$ \\
\hline$\left\langle\mathcal{O}^{J / \psi}\left({ }^{1} S_{0}^{[8]}\right)\right\rangle\left(\mathrm{GeV}^{3}\right)$ & \multicolumn{2}{|l|}{$1.8 \times 10^{-2}$} & 0 & 0 & 0.14 \\
\hline$\left\langle\mathcal{O}^{J / \psi}\left({ }^{3} P_{0}^{[8]}\right)\right\rangle\left(\mathrm{GeV}^{5}\right)$ & \multicolumn{2}{|l|}{$3.5 \times 10^{-2}$} & $4.4 \times 10^{-2}$ & $2.9 \times 10^{-2}$ & $-3.9 \times 10^{-2}$ \\
\hline$\left\langle\mathcal{O}^{\psi(2 S)}\left({ }^{3} S_{1}^{[1]}\right)\right\rangle\left(\mathrm{GeV}^{3}\right)$ & 0.76 & \multicolumn{2}{|c|}{0.67} & 0.76 & 0 \\
\hline$\left\langle\mathcal{O}^{\psi(2 S)}\left({ }^{3} S_{1}^{[8]}\right)\right\rangle\left(\mathrm{GeV}^{3}\right)$ & $3.3 \times 10^{-3}$ & \multicolumn{2}{|c|}{$3.7 \times 10^{-3}$} & $5 \times 10^{-3}$ & 0 \\
\hline$\left\langle\mathcal{O}^{\psi(2 S)}\left({ }^{1} S_{0}^{[8]}\right)\right\rangle\left(\mathrm{GeV}^{3}\right)$ & $8.0 \times 10^{-3}$ & \multicolumn{2}{|c|}{0} & 0 & 0 \\
\hline$\left\langle\mathcal{O}^{\psi(2 S)}\left({ }^{3} P_{0}^{[8]}\right)\right\rangle\left(\mathrm{GeV}^{5}\right)$ & $1.6 \times 10^{-2}$ & \multicolumn{2}{|c|}{$5.0 \times 10^{-3}$} & $1.2 \times 10^{-2}$ & 0 \\
\hline$\left\langle\mathcal{O}^{\chi_{c 0}}\left({ }^{3} S_{1}^{[8]}\right)\right\rangle\left(\mathrm{GeV}^{3}\right)$ & $1.9 \times 10^{-3}$ & \multicolumn{2}{|c|}{$1.9 \times 10^{-3}$} & $3.1 \times 10^{-3}$ & 0 \\
\hline \multirow[t]{3}{*}{$\left\langle\mathcal{O}^{\chi} \chi_{c 0}\left({ }^{3} P_{0}^{[1]}\right)\right\rangle\left(\mathrm{GeV}^{5}\right)$} & 0.11 & 9.1 & & 0.11 & 0 \\
\hline & NLO fits & & & & NLO+LP fit \\
\hline & Set 5 & Set 6 & Set 7 & Set 8 & Set 9 \\
\hline$\left\langle\mathcal{O}^{J / \psi}\left({ }^{3} S_{1}^{[1]}\right)\right\rangle\left(\mathrm{GeV}^{3}\right)$ & $1.32 \mathrm{GeV}^{3}$ & 1.16 & 1.16 & 1.16 & 1.16 \\
\hline$\left\langle\mathcal{O}^{J / \psi}\left({ }^{3} S_{1}^{[8]}\right)\right\rangle\left(\mathrm{GeV}^{3}\right)$ & $2.2 \times 10^{-3}$ & $-4.6 \times 10^{-3}$ & $1.1 \times 10^{-2}$ & $9.0 \times 10^{-3}$ & $1.1 \times 10^{-2}$ \\
\hline$\left\langle\mathcal{O}^{J / \psi}\left({ }^{1} S_{0}^{[8]}\right)\right\rangle\left(\mathrm{GeV}^{3}\right)$ & $5.0 \times 10^{-2}$ & $9.7 \times 10^{-2}$ & 0 & $1.5 \times 10^{-2}$ & $9.9 \times 10^{-2}$ \\
\hline$\left\langle\mathcal{O}^{J / \psi}\left({ }^{3} P_{0}^{[8]}\right)\right\rangle\left(\mathrm{GeV}^{5}\right)$ & $-1.6 \times 10^{-2}$ & $-2.1 \times 10^{-2}$ & $4.2 \times 10^{-2}$ & $3.4 \times 10^{-2}$ & $1.1 \times 10^{-2}$ \\
\hline$\left\langle\mathcal{O}^{\psi(2 S)}\left({ }^{3} S_{1}^{[1]}\right)\right\rangle\left(\mathrm{GeV}^{3}\right)$ & 0 & 0.76 & 0.76 & 0.76 & 0 \\
\hline$\left\langle\mathcal{O}^{\psi(2 S)}\left({ }^{3} S_{1}^{[8]}\right)\right\rangle\left(\mathrm{GeV}^{3}\right)$ & 0 & $3.4 \times 10^{-3}$ & $6.1 \times 10^{-3}$ & $1.2 \times 10^{-3}$ & 0 \\
\hline$\left\langle\mathcal{O}^{\psi(2 S)}\left({ }^{1} S_{0}^{[8]}\right)\right\rangle\left(\mathrm{GeV}^{3}\right)$ & 0 & $-1.2 \times 10^{-4}$ & 0 & $2.0 \times 10^{-2}$ & 0 \\
\hline$\left\langle\mathcal{O}^{\psi(2 S)}\left({ }^{3} P_{0}^{[8]}\right)\right\rangle\left(\mathrm{GeV}^{5}\right)$ & 0 & $9.5 \times 10^{-3}$ & $2.2 \times 10^{-2}$ & 0 & 0 \\
\hline$\left\langle\mathcal{O}^{\chi_{c 0}}\left({ }^{3} S_{1}^{[8]}\right)\right\rangle\left(\mathrm{GeV}^{3}\right)$ & 0 & $2.2 \times 10^{-3}$ & $2.2 \times 10^{-3}$ & $2.2 \times 10^{-3}$ & 0 \\
\hline$\left\langle\mathcal{O}^{\chi c 0}\left({ }^{3} P_{0}^{[1]}\right)\right\rangle\left(\mathrm{GeV}^{5}\right)$ & 0 & 0.11 & 0.11 & 0.11 & 0 \\
\hline$P_{T}(J / \psi)$ region & $<30 \mathrm{GeV}$ & $>7 \mathrm{GeV}$ & $>7 \mathrm{GeV}$ & $>7 \mathrm{GeV}$ & $>10 \mathrm{GeV}$ \\
\hline
\end{tabular}

i.e. only to the ATLAS fiducial region for our forthcoming discussion of double $J / \psi$ production.

5. The two sets denoted sets 7 and 8 in Table 3 are two extreme cases of the PKU fit $[69,70]$ after including the constraints from LHC $\eta_{c}$ data $[69,70]$. They supersede the fits including the $P_{T}(J / \psi)>7 \mathrm{GeV}$ hadroproduction data described in Refs. [77,78]. These LDME sets cannot reproduce the CDF polarisation measurement [79]—-like all the other sets in fact—and are not applicable to $P_{T}(J / \psi)<7 \mathrm{GeV}$. Both sets should only be used to di- $\psi$ production in the ATLAS fiducial region.

6. The NLO+LP fit of Ref. [71] —and its update [80] —has been presented by its authors as the only fit able to reproduce the $J / \psi$ data (both yields and polarisations) above $10 \mathrm{GeV}$ after including the LP fragmentation contributions on top of the NLO calculations. However, it does not yield the correct $\eta_{c}$ cross section in the same $P_{T}$ region under the heavy-quark spin symmetry. As what concerns predictions for double $J / \psi$ production, it is marginally applicable only in the ATLAS fiducial region with $P_{T}(J / \psi)>8.5 \mathrm{GeV}$ instead of $10 \mathrm{GeV}$.

Since we aim at a comprehensive analysis, we have considered all of the nine sets listed in Table 3 to show how strongly the $\mathrm{CO}$ contributions depend on the available $\mathrm{CO}$ LDMEs. We should, however, recall in the discussion what we believe to be the region of applicability in $P_{T}(J / \psi)$ for the $\mathrm{NLO}(+\mathrm{LP})$ fits.

\subsection{Numerical results}

In this section, we will present our numerical results with the LO CO channels summed to the pure $\mathrm{NLO}^{\star} \mathrm{CS}$ channel ${ }^{3} S_{1}^{[1]}+{ }^{3} S_{1}^{[1]}$. Although the CS LDMEs $\left\langle\mathcal{O}^{J / \psi}\left({ }^{3} S_{1}^{[1]}\right)\right\rangle$ and $\left\langle\mathcal{O}^{\psi(2 S)}\left({ }^{3} S_{1}^{[1]}\right)\right\rangle$ vary from set to set in Table 3, we will 




(a) Set 1

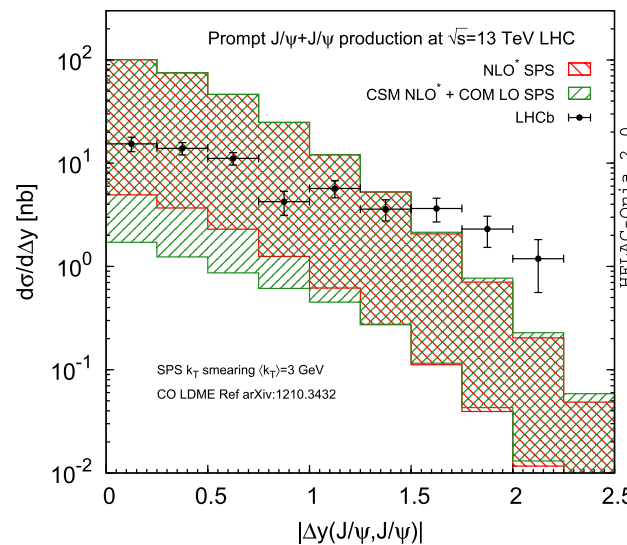

(d) Set 4

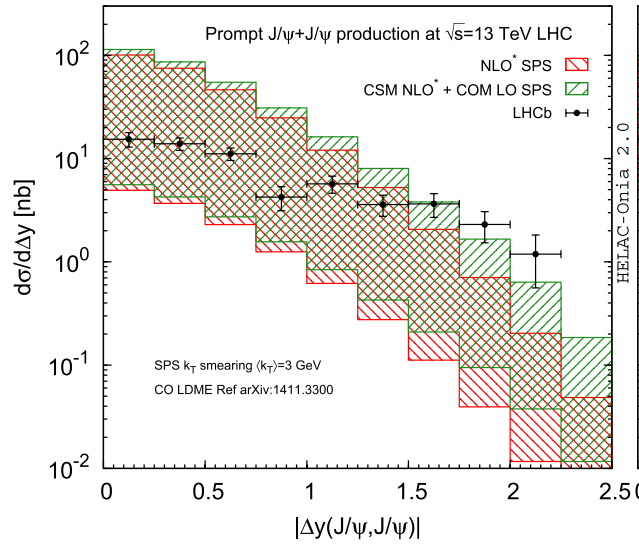

(g) Set 7



(b) Set 2

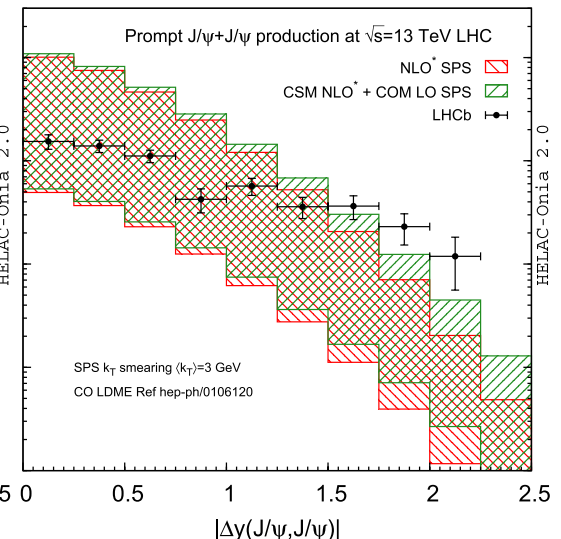

(c) Set 3

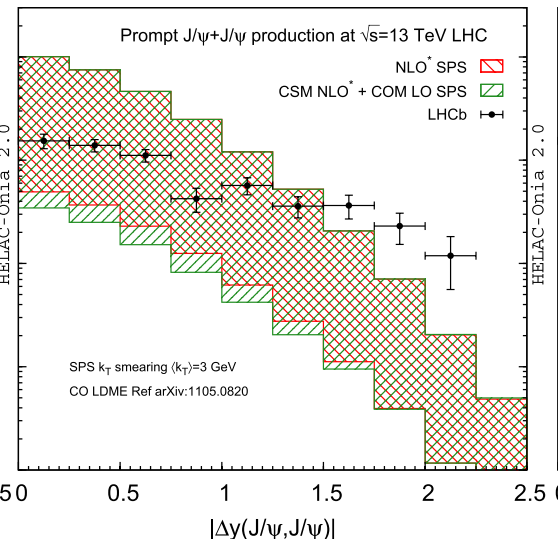

(e) Set 5

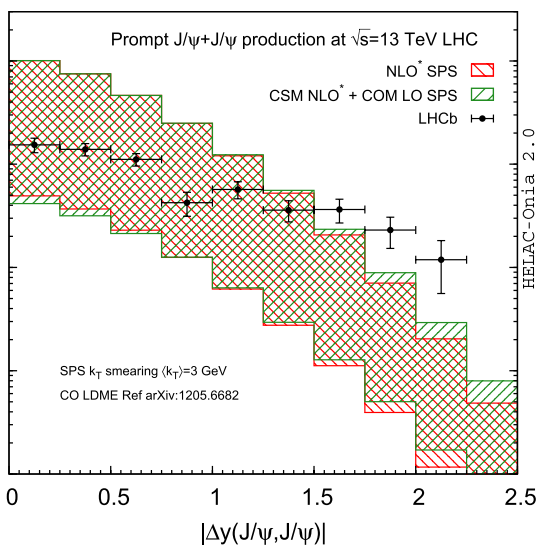

(f) Set 6

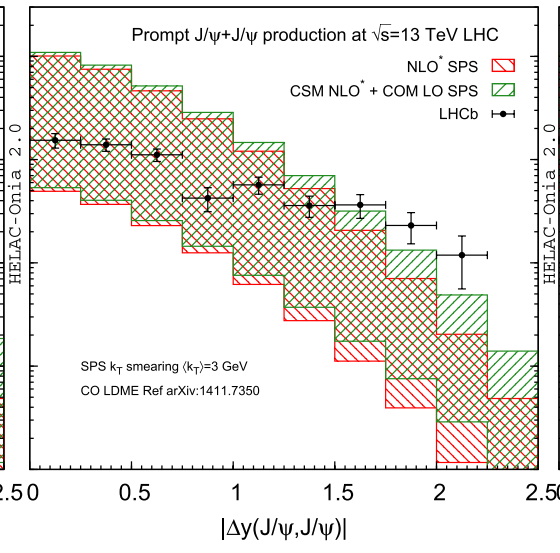

(h) Set 8

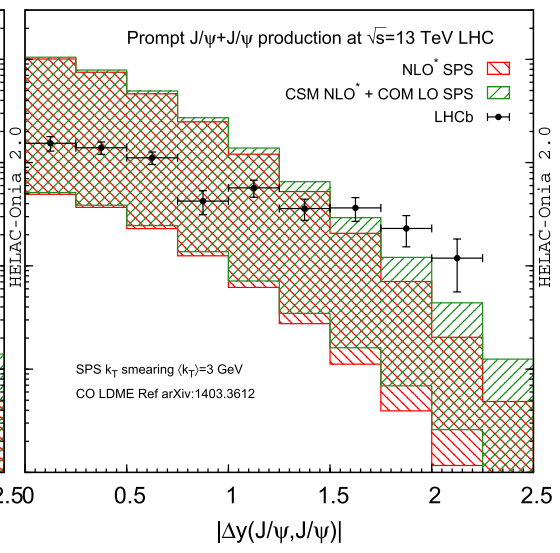

(i) Set 9

Fig. $4 \Delta y(J / \psi, J / \psi)$ distributions in $\mathrm{NLO}^{\star} \mathrm{CS}$ and LO CO via SPS within LHCb $\sqrt{s}=13 \mathrm{TeV}$ acceptance [23]

fix these values for the $\mathrm{NLO}^{\star} \mathrm{CS}$ channel ${ }^{3} S_{1}^{[1]}+{ }^{3} S_{1}^{[1]}$ to those used in Sect. 3.2. The uncertainty from these LDMEs is systematically subdominant compared to the scale uncertainty. All the feed-down contributions are properly taken into account as well.

\subsubsection{LHCb data at $\sqrt{s}=13 \mathrm{TeV}$}

We start our discussion with the LHCb acceptance [23], where the $P_{T}(J / \psi)$ can be as low as zero. We have compared the CSM NLO ${ }^{\star}+\mathrm{COM}$ LO SPS (the green bands) with 


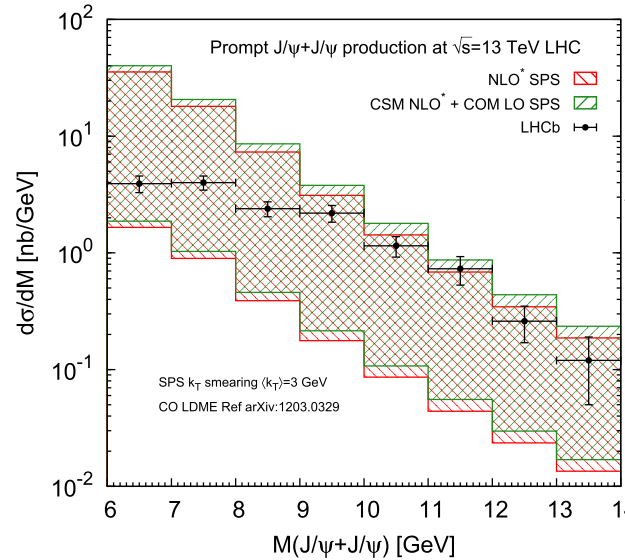

(a) Set 1

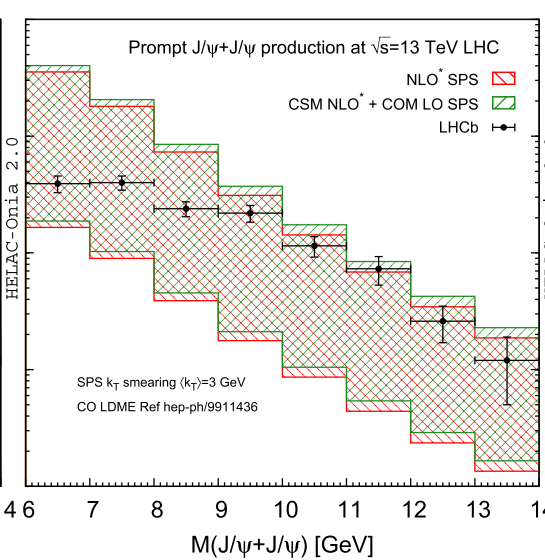

(b) Set 2



(c) Set 3

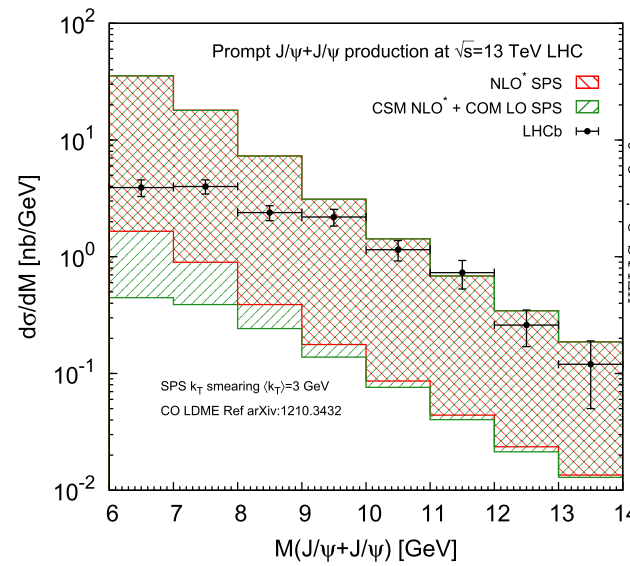

(d) Set 4

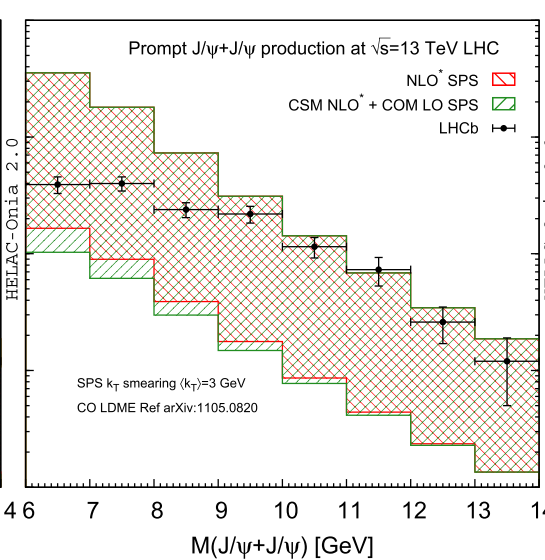

(e) Set 5

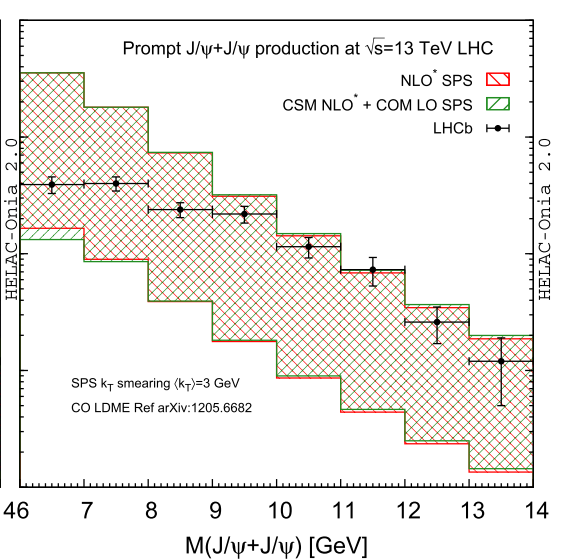

(f) Set 6

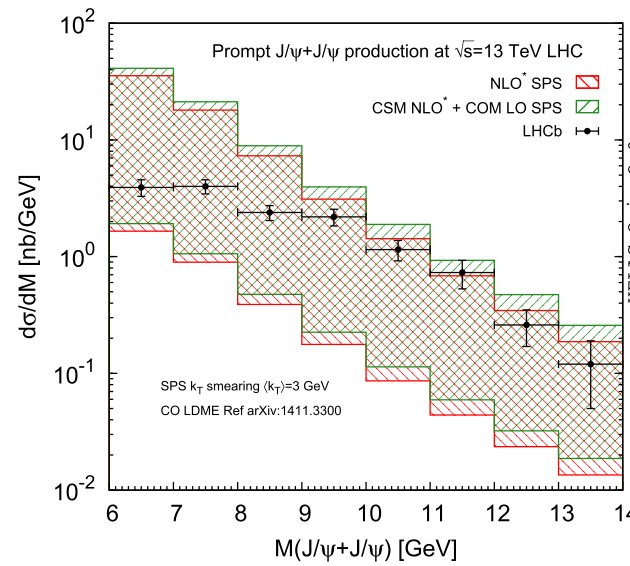

(g) Set 7

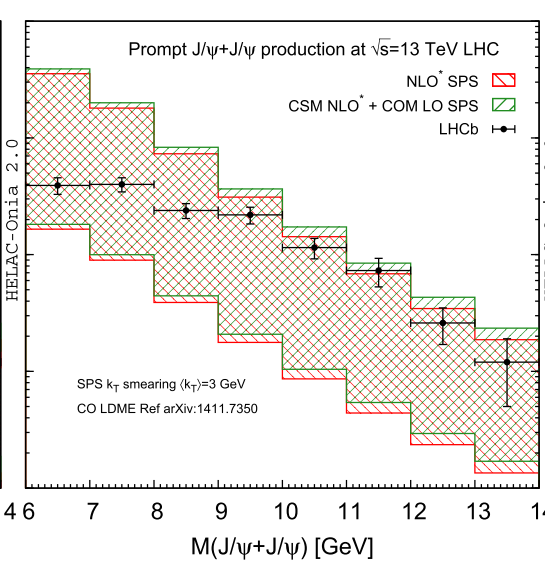

(h) Set 8

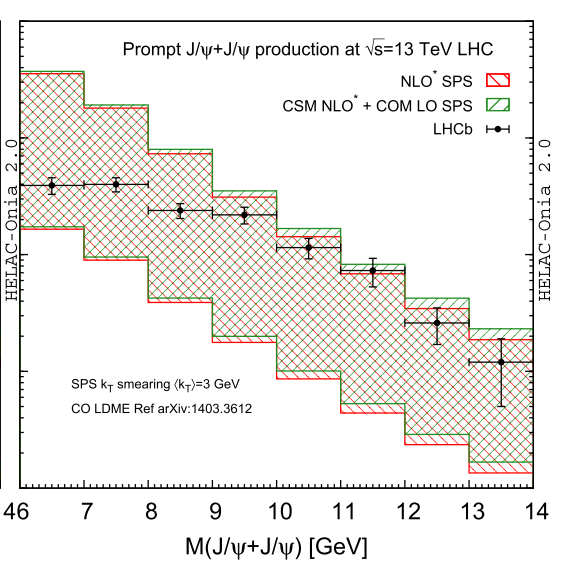

(i) Set 9

Fig. $5 M(J / \psi+J / \psi)$ distributions in $\mathrm{NLO}^{\star} \mathrm{CS}$ and LO CO via SPS within LHCb $\sqrt{s}=13 \mathrm{TeV}$ acceptance [23]

the data in Fig. 4 for the $\Delta y(J / \psi, J / \psi)$ distribution and in Fig. 5 for the invariant mass of the pair $M(J / \psi+J / \psi)$ distribution. Like we have found for the CS LI contributions, the $\mathrm{CO}$ contributions are not relevant in the invariant-mass distribution of LHCb. They start to be slightly visible in the tail of the $\Delta y(J / \psi, J / \psi)$ distribution.
This observation, however, very much depends on the set of CO LDMEs used. In particular, the only plausible set, i.e. set 5, in the small $P_{T}(J / \psi)$ region does not yield any significant contribution to the cross section. It also seems clear that none of the sets can fully account for the discrepancy between SPS and LHCb data in the last bins of $\frac{d \sigma}{d \Delta y}$. Addi- 


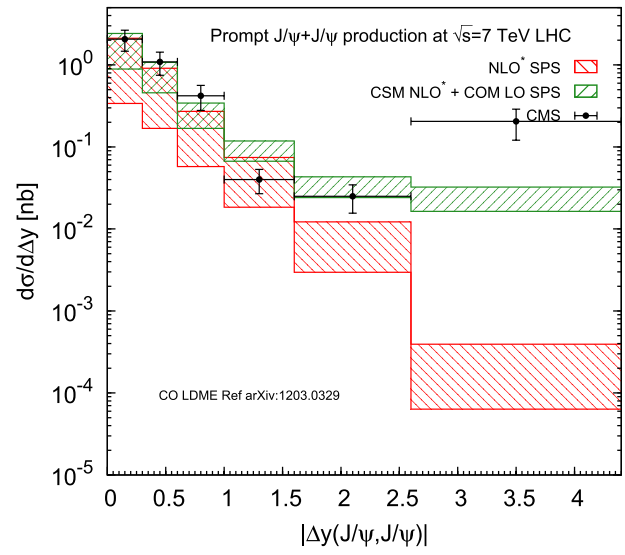

(a) Set 1

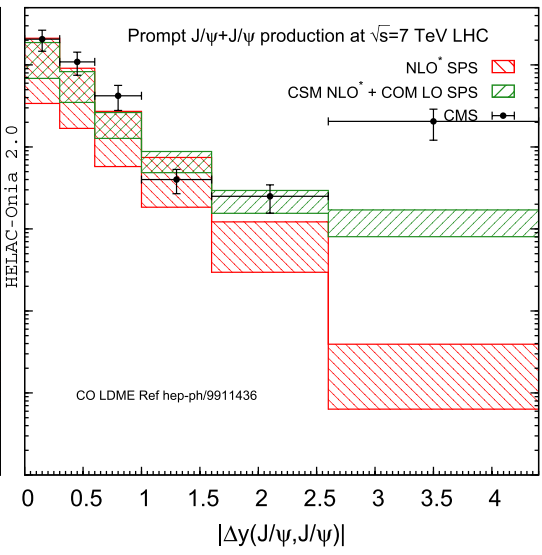

(b) Set 2

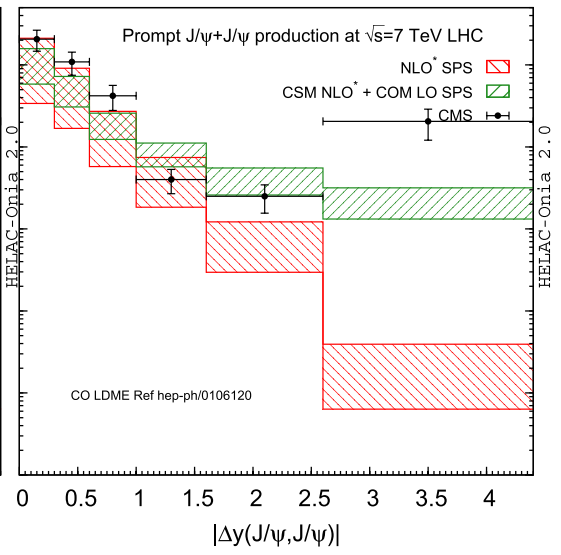

(c) Set 3

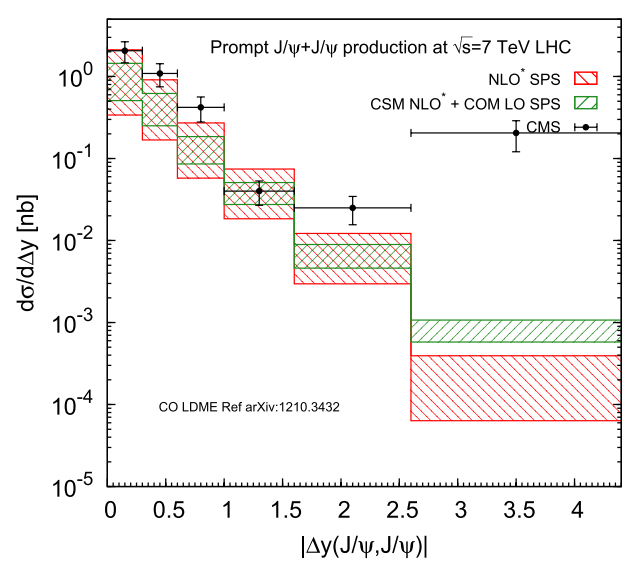

(d) Set 4

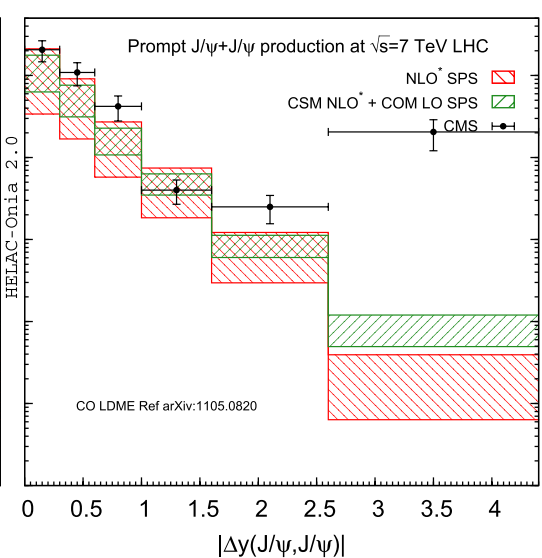

(e) Set 5

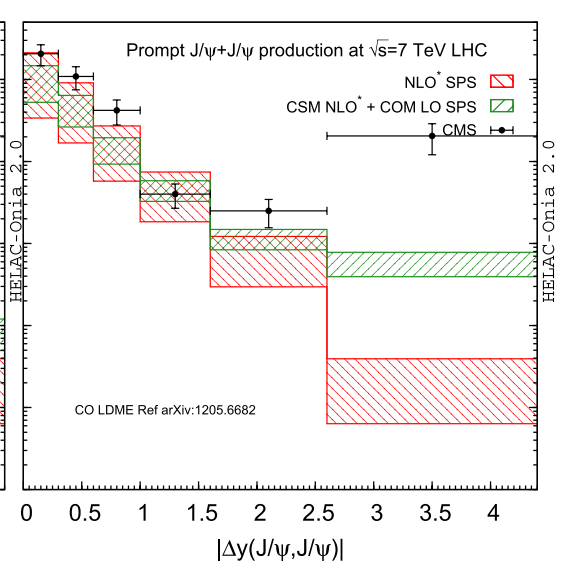

(f) Set 6



(g) Set 7

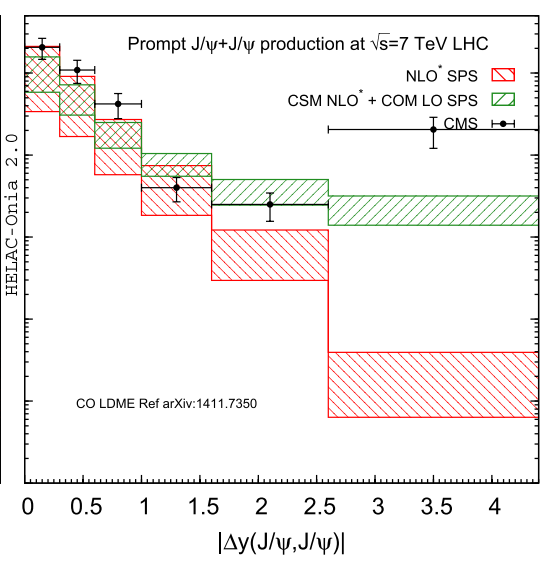

(h) Set 8

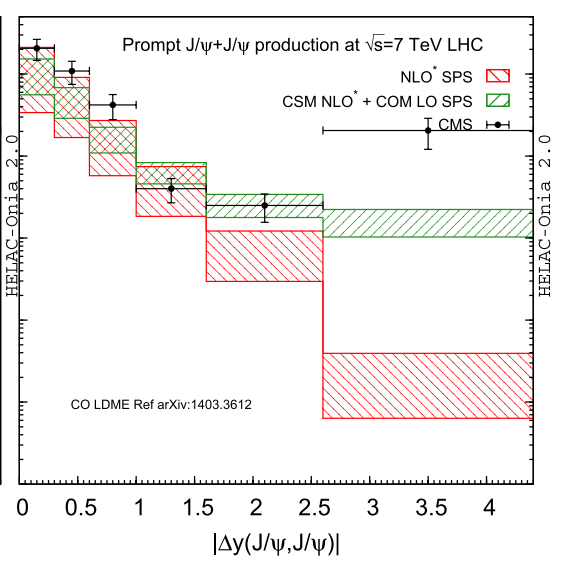

(i) Set 9

Fig. $6 \Delta y(J / \psi, J / \psi)$ distributions in $\mathrm{NLO}^{\star} \mathrm{CS}$ and LO CO via SPS within CMS $\sqrt{s}=7 \mathrm{TeV}$ acceptance [21]

tional plots for the comparisons between $\mathrm{CS} \mathrm{NLO}^{\star}+\mathrm{CO} \mathrm{LO}$ SPS and data can be found in Appendix B.2. The impact of the $\mathrm{CO}$ contributions on these additional distributions is in general minor.

\subsubsection{CMS data at $\sqrt{s}=7 \mathrm{TeV}$}

The events analysed by CMS have larger $P_{T}(J / \psi)$, above $4.5 \mathrm{GeV}$ to $6.5 \mathrm{GeV}$, depending on the rapidity. In this region, the only applicable NLO fit is still set 5 taken from Ref. [67]. 




(a) Set 1

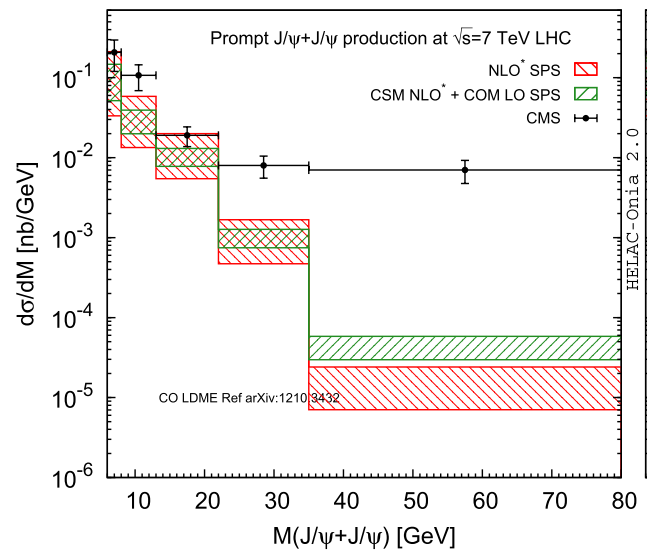

(d) Set 4

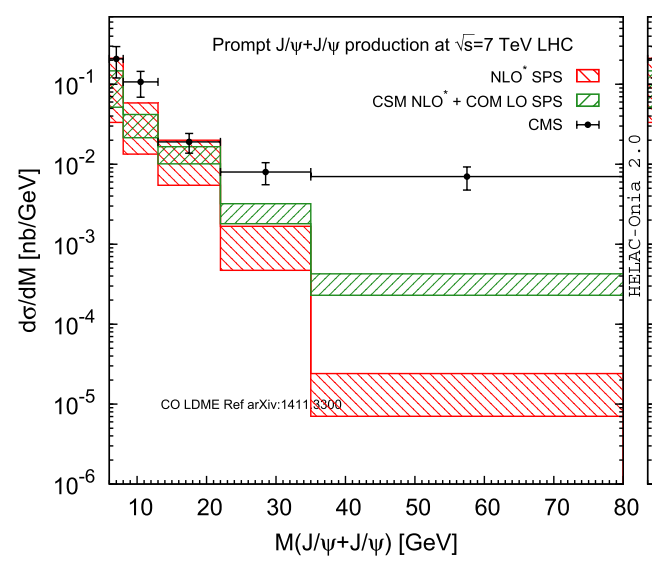

(g) Set 7



(b) Set 2

(c) Set 3

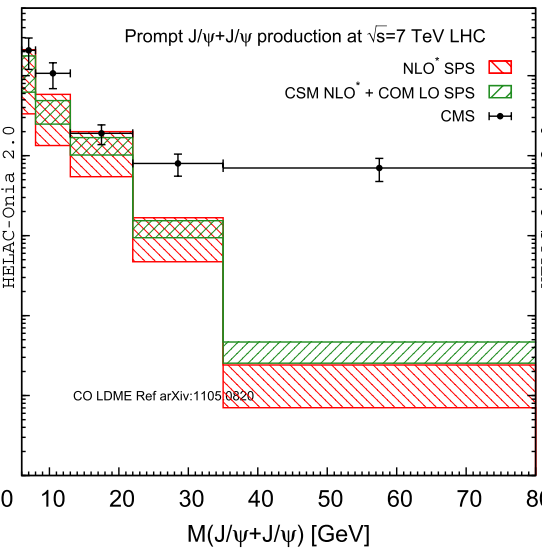

(e) Set 5

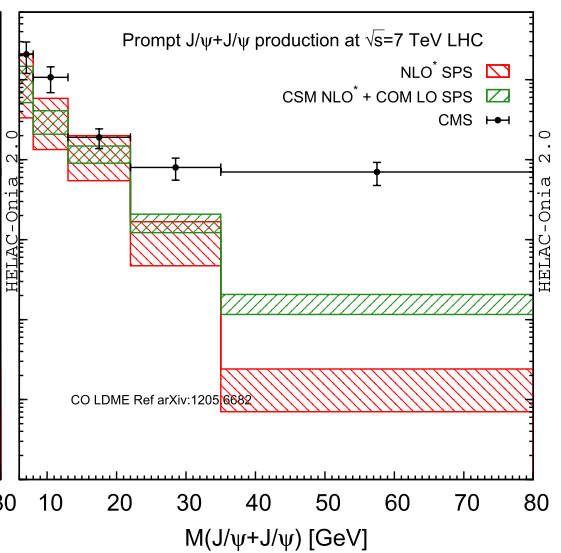

(f) Set 6

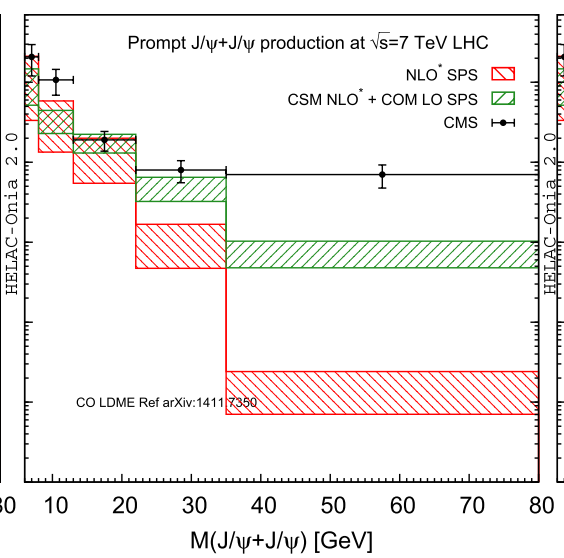

(h) Set 8

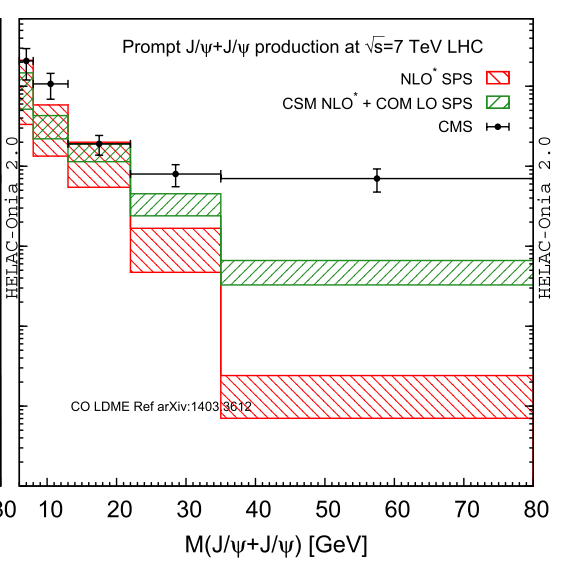

(i) Set 9

Fig. $7 M(J / \psi, J / \psi)$ distributions in $\mathrm{NLO}^{\star} \mathrm{CS}$ and LO CO via SPS within CMS $\sqrt{s}=7 \mathrm{TeV}$ acceptance [21]

As opposed to the conclusion made in Ref. [40], the CO SPS contribution is either much suppressed compared to the CS SPS contributions or much smaller than the experimental data as shown in Figs. 6 and 7. Given that the LO fits (like that used in Ref. [40] (i.e. set 2)) are not plausible any more and that the only applicable fit is the NLO fit given by set 5, we draw the conclusion that our extraction of DPS in Ref. [37] — made by neglecting the CO contributions-is still sound, which actually has been shown to be consistent with the ATLAS measurement thanks to a completely different method to disentangle the DPS from the SPS contributions and in a different kinematical region. 




(a) Set 1



(b) Set 2

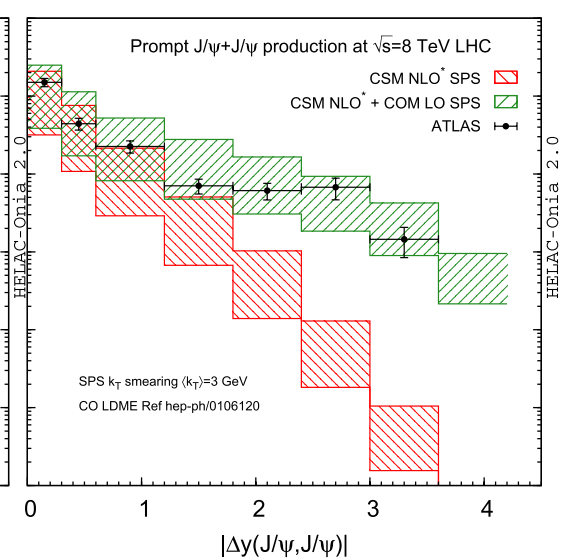

(c) Set 3

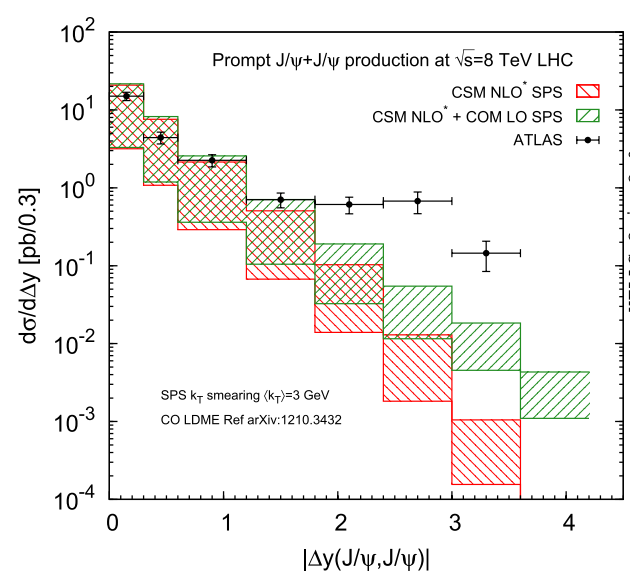

(d) Set 4

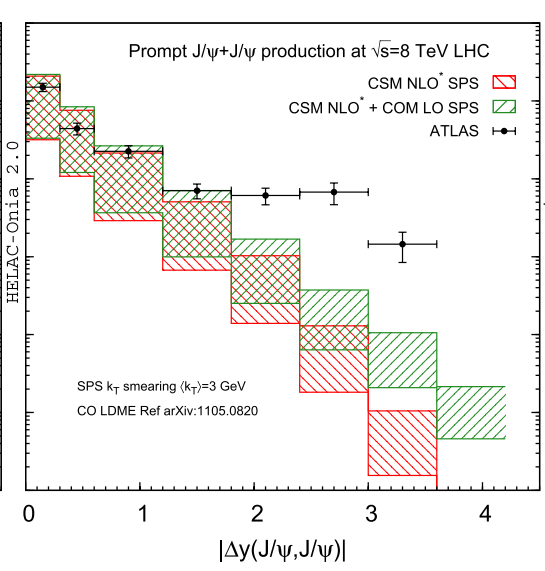

(e) Set 5

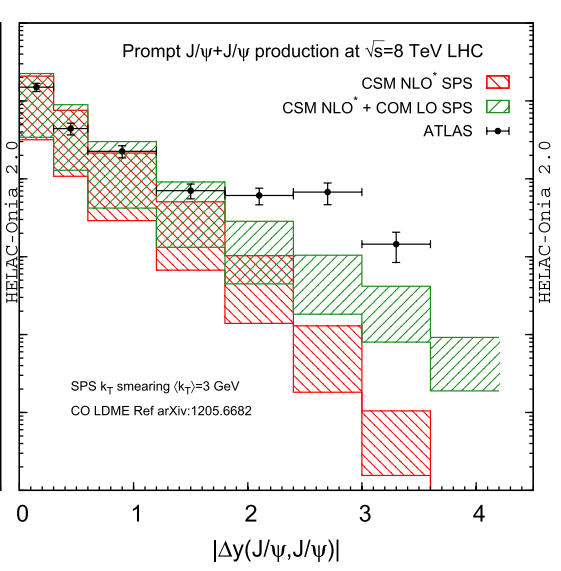

(f) Set 6

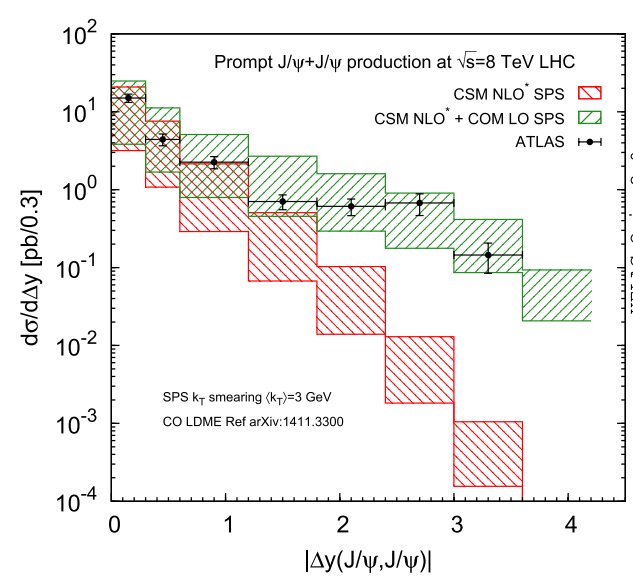

(g) Set 7

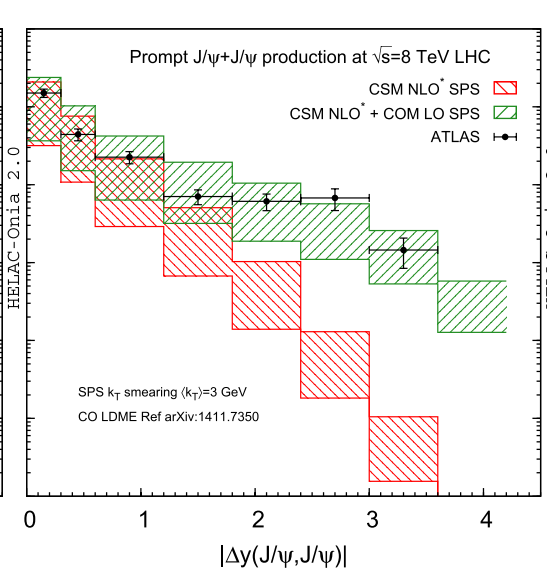

(h) Set 8

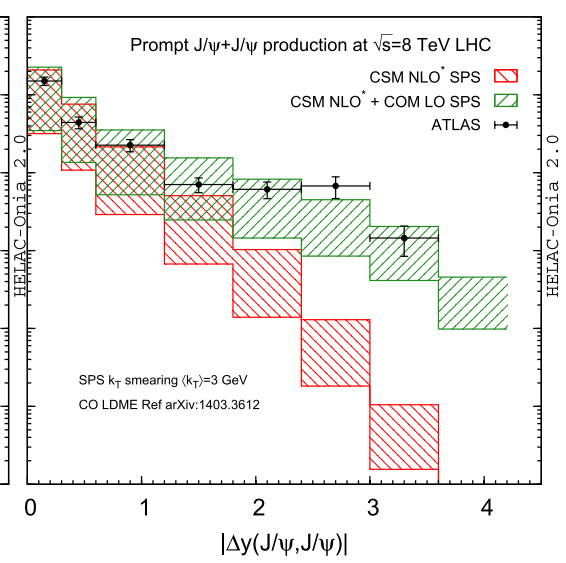

(i) Set 9

Fig. $8 \Delta y(J / \psi, J / \psi)$ distributions in $\mathrm{NLO}^{\star} \mathrm{CS}$ and LO CO via SPS within ATLAS $\sqrt{s}=8 \mathrm{TeV}$ acceptance [22]

\subsubsection{ATLAS data at $\sqrt{s}=8 \mathrm{TeV}$}

The transverse-momentum cut on single $J / \psi$ is largest in the ATLAS data sample with selected events satisfying $P_{T}(J / \psi)>8.5 \mathrm{GeV}$. This leaves the LDME sets $5-8$ as pos- sible good fits. ATLAS used a 2D $(|\Delta y(J / \psi, J / \psi)|, \Delta \phi(J /$ $\psi, J / \psi)$ ) data-driven template fit to separate SPS and DPS events [22]. The control region used to determine the normalisation of DPS is $(|\Delta y(J / \psi, J / \psi)| \geq 1.8, \Delta \phi(J / \psi, J / \psi) \leq$ $\left.\frac{\pi}{2}\right)$. The requirement of $\Delta \phi(J / \psi, J / \psi) \leq \frac{\pi}{2}$ will signif- 


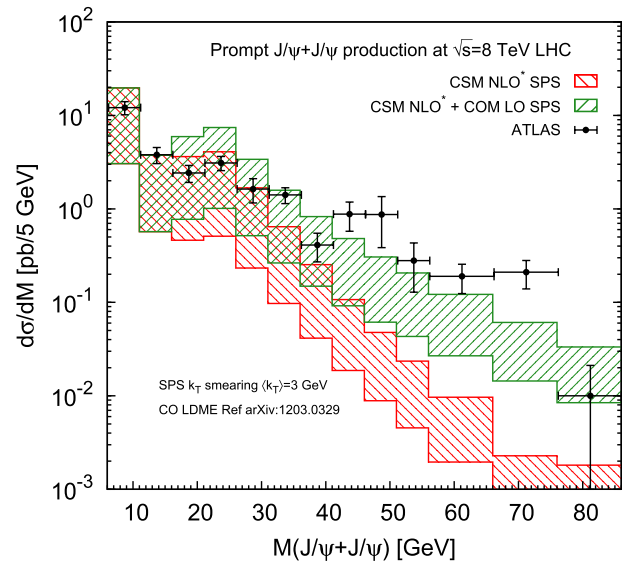

(a) Set 1

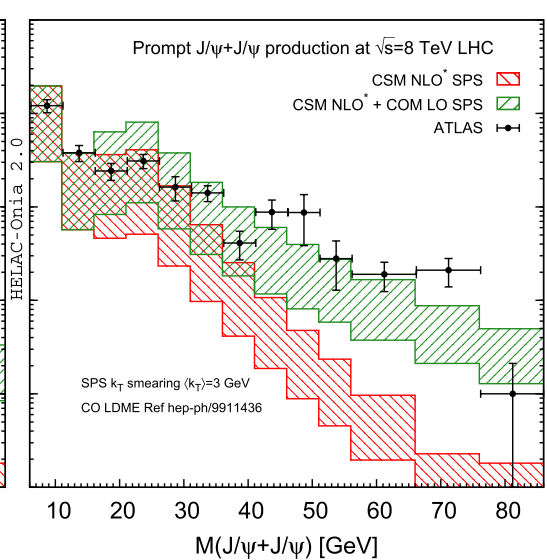

(b) Set 2



(c) Set 3

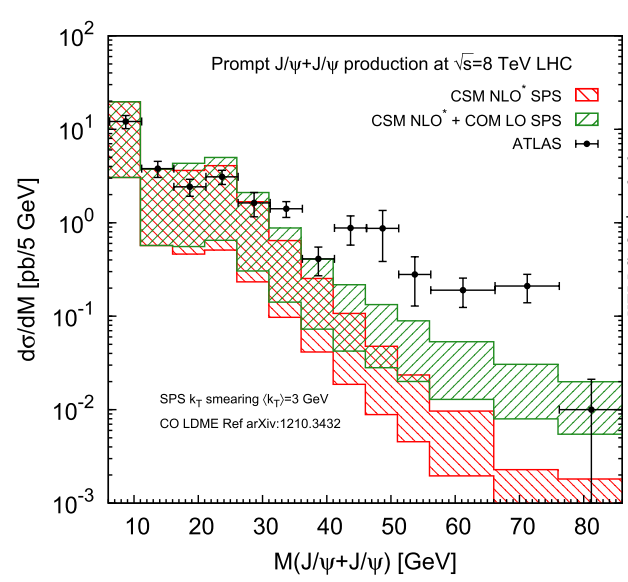

(d) Set 4

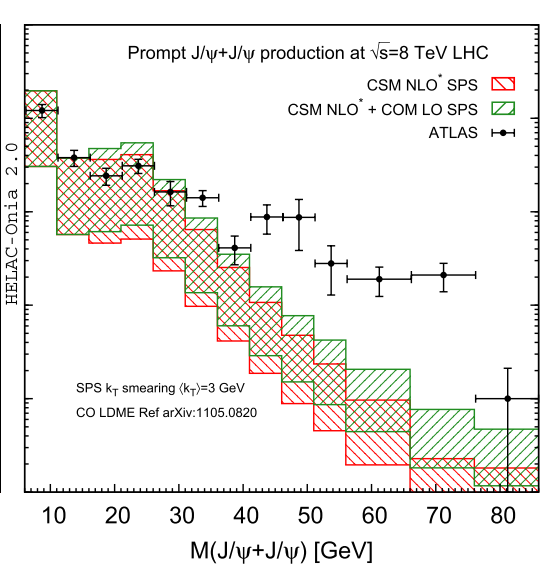

(e) Set 5

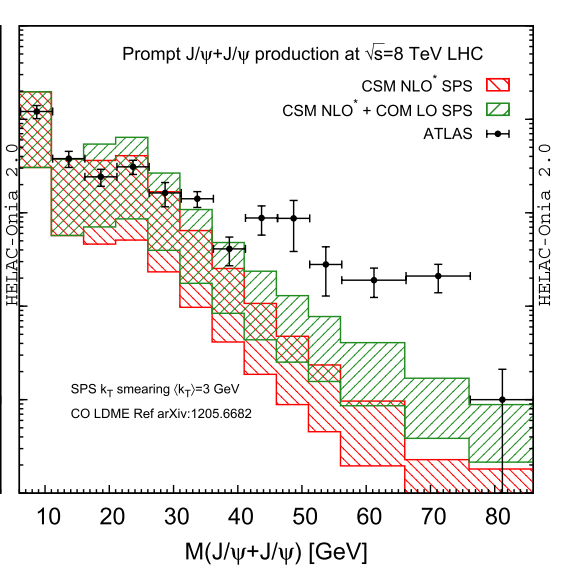

(f) Set 6

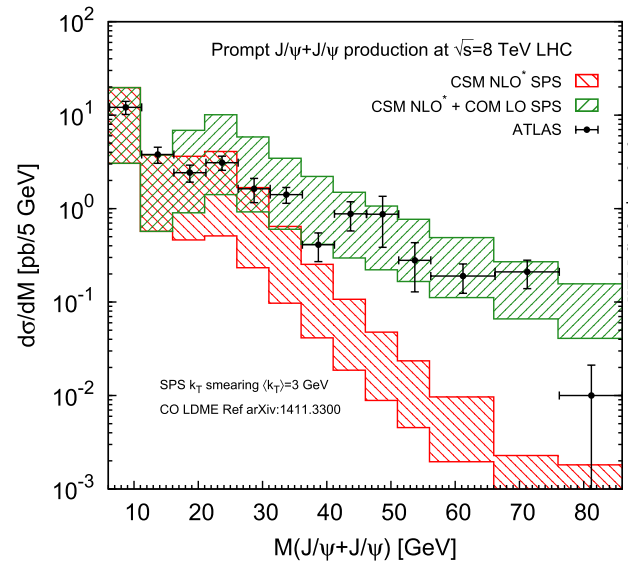

(g) Set 7

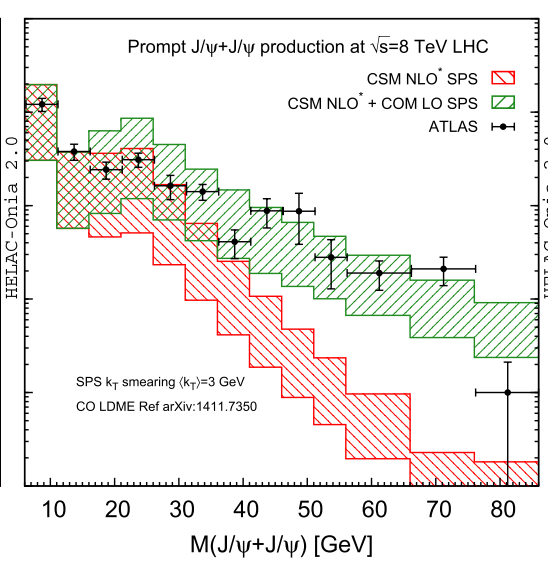

(h) Set 8



(i) Set 9

Fig. $9 M(J / \psi, J / \psi)$ distributions in NLO ${ }^{\star}$ CS and LO CO via SPS within ATLAS $\sqrt{s}=8 \mathrm{TeV}$ acceptance [22]

icantly reduce the CO fraction at large $|\Delta y(J / \psi, J / \psi)|$. The $t$-channel gluon exchange diagrams mainly make the two $J / \psi$ recoiling against each other. It thus populates the region where $\Delta \phi(J / \psi, J / \psi) \rightarrow \pi$. The simultaneous cuts on $|\Delta y(J / \psi, J / \psi)|$ and $\Delta \phi(J / \psi, J / \psi)$ ensure that the DPS extraction in Ref. [22] is reliable but for the low statistics in the control region. From Figs. 8 and 9, one sees that the $\mathrm{CO}$ yields predicted with the sets 7 and 8 agree reasonably well with the data at large $M(J / \psi, J / \psi)$ and $|\Delta y(J / \psi, J / \psi)|$ with a slight overestimation in the middle of the corresponding distributions. The sets 5 and 6 , however, do not agree with the data. Strong conclusions about the relevance of $\mathrm{CO}$ 
transitions in these regions would thus probably be premature in the absence of a complete NLO study and the disparate values of the existing CO LDMEs.

\section{Conclusions}

We have examined two SPS production mechanisms for di$J / \psi$ production at the LHC, which can be relevant in the control region used to determine the DPS. These are the partial LI CS contributions at $\mathcal{O}\left(\alpha_{S}^{6}\right)$ and the LO CO contributions at $\mathcal{O}\left(\alpha_{S}^{4}\right)$. We have also extensively compared our new SPS calculations with the existing LHC data. Our study indeed shows that the LI corrections can enhance the NLO ${ }^{\star}$ SPS cross section at large $|\Delta y(J / \psi, J / \psi)|$ and large invariant mass $M(J / \psi+J / \psi)$. However, they are not sufficient to explain the discrepancy between SPS theoretical results and the LHC data in these regions. The inclusion of the DPS in the predictions is still crucial to accounting for the measurements.

On the other hand, the relevance of the $\mathrm{CO}$ contributions in the SPS yield strongly depends on the considered LDME set, thus with a very low predictive power-given the current status of understanding of the COM. It is in any case confined to the large $|\Delta y(J / \psi, J / \psi)|$ region. We anyhow conclude that the $\mathrm{CO}$ contributions can only be important when compared to the ATLAS data but that the ATLAS DPS extraction via a 2D data-driven fit is very likely free of any bias due to a possibly underestimated $\mathrm{CO}$ contribution in their control region. Such a conclusion is backed up by studies [81,82] made in the colour-evaporation model which offers a complementary framework to study the impact of CO transitions.

Acknowledgements The work of JPL, HSS, YJZ is supported in part by CNRS via the LIA FCPPL. JPL is supported in part by the TMD@NLO IN2P3 project. The work of HSS is supported by the ILP Labex (ANR-11-IDEX-0004-02, ANR-10-LABX-63). YJZ is supported by the National Natural Science Foundation of China (Grants No. 11722539). NY was supported by the JSPS Postdoctoral Fellowships for Research Abroad.

Data Availability Statement This manuscript has no associated data or the data will not be deposited. [Authors' comment: All data (numbers and plots) generated in our study have been included in this paper. We do not have additional data to show.]

Open Access This article is licensed under a Creative Commons Attribution 4.0 International License, which permits use, sharing, adaptation, distribution and reproduction in any medium or format, as long as you give appropriate credit to the original author(s) and the source, provide a link to the Creative Commons licence, and indicate if changes were made. The images or other third party material in this article are included in the article's Creative Commons licence, unless indicated otherwise in a credit line to the material. If material is not included in the article's Creative Commons licence and your intended use is not permitted by statutory regulation or exceeds the permitted use, you will need to obtain permission directly from the copyright holder. To view a copy of this licence, visit http://creativecomm ons.org/licenses/by/4.0/.

Funded by $\mathrm{SCOAP}^{3}$.

\section{Appendix A CO predictions with a hybrid LDME set}

In order to exhaust other possibilities, we take a hybrid LDME set by using the CO LDMEs of $J / \psi$ from the feeddown subtracted fit in Ref. [67] and the CO LDMEs of $\chi_{c J}$ and $\psi(2 S)$ from Ref. [69]. Their values are listed in Table 4. However, one should be cautious to use this hybrid set. Since the $P_{T}(J / \psi)$ range of the experimental data in a CO LDME fit is so crucial (see e.g. Table 3 of Ref. [69]), such a hybrid LDME set is never tested even in single prompt $J / \psi$ production. The largest applicable range of such a set is $7<$ $P_{T}(J / \psi) / \mathrm{GeV}<30$. Therefore, in principle, the di- $J / \psi$ predictions with this hybrid set are valid only for the ATLAS kinematical acceptance. Nevertheless, we have shown the three $|\Delta y(J / \psi, J / \psi)|$ distributions in comparison with CMS, ATLAS and LHCb data in Fig. 10 under this set.

\section{Appendix B Additional plots: further comparisons with data}

This appendix gathers additional plots of comparisons between our SPS calculations and experimental data collected by the ATLAS, CMS and LHCb experiments.

\section{Appendix B.1 Further comparisons with theory including} partial CS LI corrections

We compare below our SPS CS NLO $+\mathrm{LI}$ calculation to the experimental data for other observables than the rapidity difference and the invariant mass. The transverse-momentum distributions of the pair $P_{T}(J / \psi+J / \psi)$ are shown in Fig. 11 (CMS), in the left panel of Fig. 12 (ATLAS) and in the topright panel of Fig. 13 (LHCb). The $\mathrm{NLO}^{\star}+\mathrm{LI}$ SPS green bands almost overlay at the red bands (NLO ${ }^{\star}$ SPS), which
Table 4 The values of LDMEs we used from Ref. [67] and Ref. [69]

\begin{tabular}{lllll}
\hline$\left\langle\mathcal{O}^{J / \psi}\left({ }^{3} S_{1}^{[1]}\right)\right\rangle$ & $\left\langle\mathcal{O}^{J / \psi}\left({ }^{3} S_{1}^{[8]}\right)\right\rangle$ & $\left\langle\mathcal{O}^{J / \psi}\left({ }^{1} S_{0}^{[8]}\right)\right\rangle$ & $\left\langle\mathcal{O}^{J / \psi}\left({ }^{3} P_{0}^{[8]}\right)\right\rangle$ & $\left\langle\mathcal{O}^{\psi(2 S)}\left({ }^{3} S_{1}^{[1]}\right)\right\rangle$ \\
$1.32 \mathrm{GeV}^{3}$ & $1.68 \cdot 10^{-3} \mathrm{GeV}^{3}$ & $3.04 \cdot 10^{-2} \mathrm{GeV}^{3}$ & $-9.08 \cdot 10^{-3} \mathrm{GeV}^{5}$ & $0.76 \mathrm{GeV}^{3}$ \\
$\left\langle\mathcal{O}^{\psi(2 S)}\left({ }^{3} S_{1}^{[8]}\right)\right\rangle$ & $\left\langle\mathcal{O}^{\psi(2 S)}\left({ }^{1} S_{0}^{[8]}\right)\right\rangle$ & $\left\langle\mathcal{O}^{\psi(2 S)}\left({ }^{3} P_{0}^{[8]}\right)\right\rangle$ & $\left\langle\mathcal{O}^{\chi}{ }_{c 0}\left({ }^{3} S_{1}^{[8]}\right)\right\rangle$ & $\left\langle\mathcal{O} \chi_{c 0}\left({ }^{3} P_{0}^{[1]}\right)\right\rangle$ \\
$1.2 \cdot 10^{-3} \mathrm{GeV}^{3}$ & $2 \cdot 10^{-2} \mathrm{GeV}^{3}$ & 0 & $2.2 \cdot 10^{-3} \mathrm{GeV}^{3}$ & $1.07 \cdot 10^{-1} \mathrm{GeV}^{5}$ \\
\hline
\end{tabular}




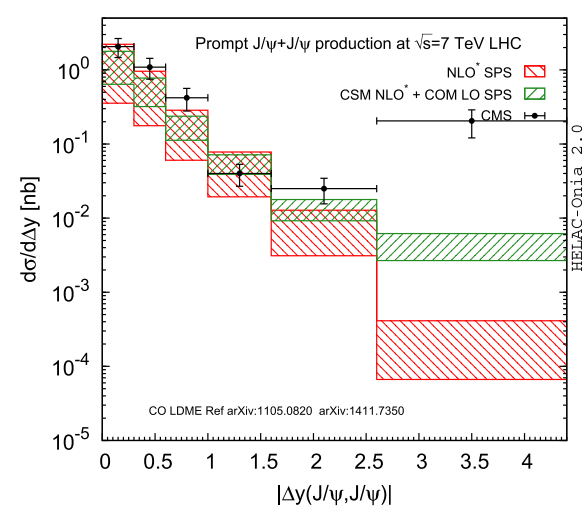

(a) $\mathrm{CMS} \sqrt{s}=7 \mathrm{TeV}$



(b) ATLAS $\sqrt{s}=8 \mathrm{TeV}$

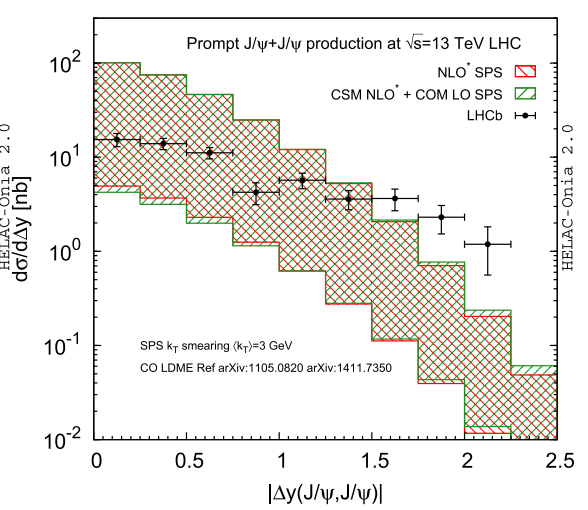

(c) $\mathrm{LHCb} \sqrt{s}=13 \mathrm{TeV}$

Fig. $10|\Delta y(J / \psi, J / \psi)|$ distributions via SPS NLO ${ }^{\star}$ CS and LO CO in the CMS, ATLAS, LHCb acceptances at $\sqrt{s}=7,8,13 \mathrm{TeV}$, respectively, where we have used the hybrid CO LDME set

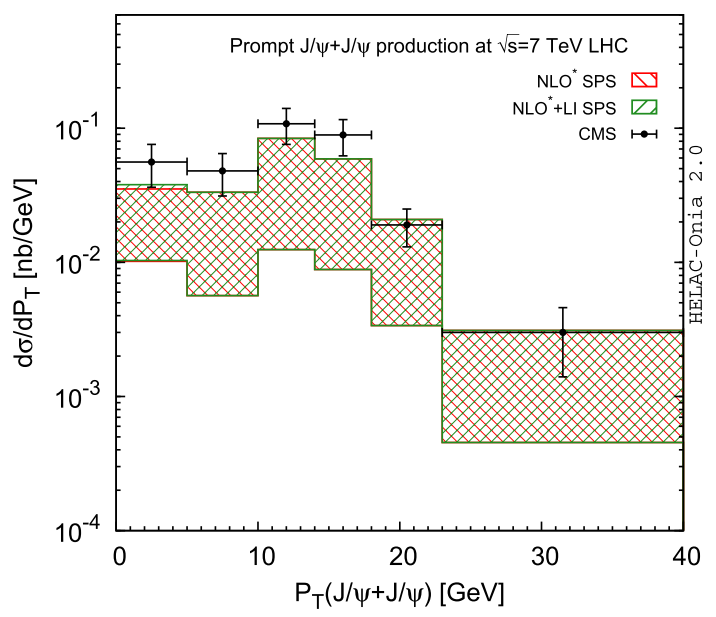

Fig. $11 P_{T}(J / \psi+J / \psi)$ distribution for CS SPS in the CMS acceptance at $\sqrt{s}=7 \mathrm{TeV}[21]$

implies that these LI corrections are not important for these distributions. It is interesting to note that the initial $k_{T^{-}}$- smearing effect is important in the low $P_{T}(J / \psi+J / \psi)$ region, which illustrates that this distribution is indeed ideal to extract the transverse-momentum dependent information from the colliding partons inside the protons

Appendix B.2 Further comparisons with theory including $\mathrm{CO}$ contributions

Further comparisons between CS NLO ${ }^{\star}+\mathrm{CO}$ LO SPS results and $\mathrm{LHCb}$ data are shown in Fig. 14 for $P_{T}(J / \psi+J / \psi)$, Fig. 15 for $\Delta \phi(J / \psi, J / \psi)$, Fig. 16 for $P_{T}(J / \psi)$, Fig. 17 for $y(J / \psi)$, Fig. 18 for $y(J / \psi+J / \psi)$ and Fig. 19 for $A_{T}(J / \psi, J / \psi)$, respectively. The inclusion of CO channels only slightly changes the corresponding predicted distributions of the SPS yield regardless of the set of LDMEs. Similar conclusions can be drawn for $P_{T}(J / \psi+J / \psi)$ and $\Delta \phi(J / \psi, J / \psi)$ distributions in the CMS and ATLAS acceptances, which is clearly seen in Figs. 20, 21 and 22. 
Fig. $12 P_{T}(J / \psi+J / \psi)$ (top) and $\Delta \phi(J / \psi, J / \psi)$ (bottom) distributions for CS SPS in ATLAS $\sqrt{s}=8 \mathrm{TeV}$ acceptance [22]


Fig. $13 A_{T}(J / \psi, J / \psi)$ (top left), $P_{T}(J / \psi)$ (top middle), $P_{T}(J / \psi+J / \psi)$ (top right), $\Delta \phi(J / \psi, J / \psi)$ (bottom left), $y(J / \psi)$ (bottom middle) and $y(J / \psi+J / \psi)$ (bottom right) distributions for di- $J / \psi$ production via CS SPS within LHCb $\sqrt{s}=13 \mathrm{TeV}$ acceptance [23] 


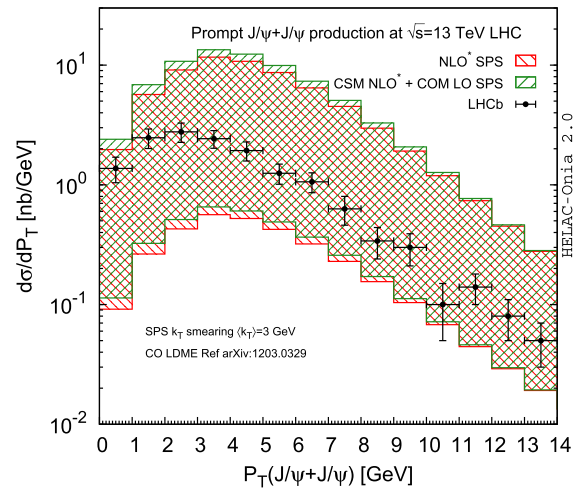

(a) Set 1

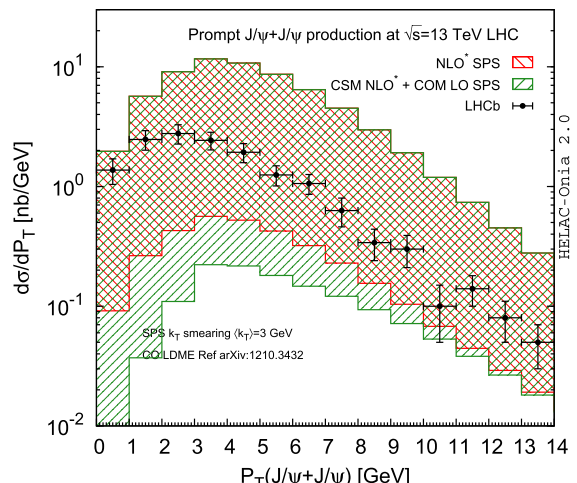

(d) Set 4

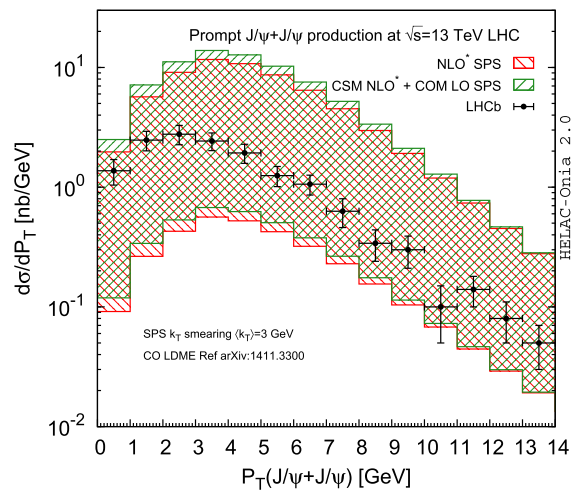

(g) Set 7

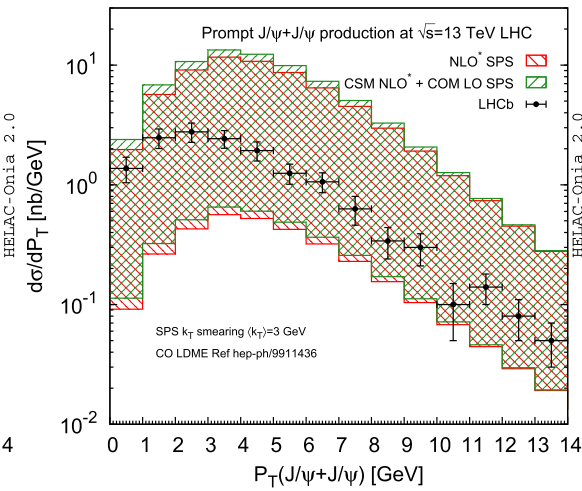

(b) Set 2



(e) Set 5

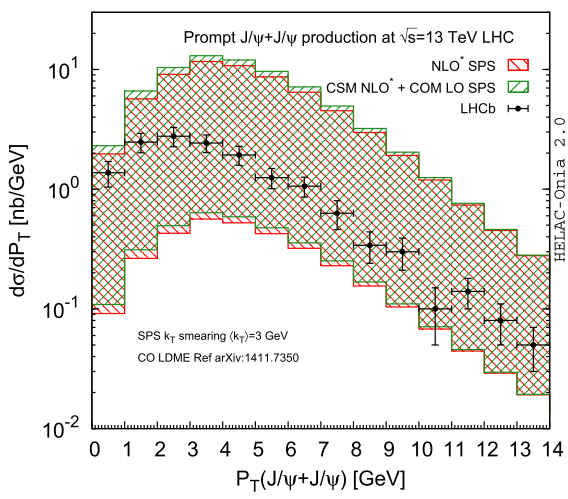

(h) Set 8



(c) Set 3

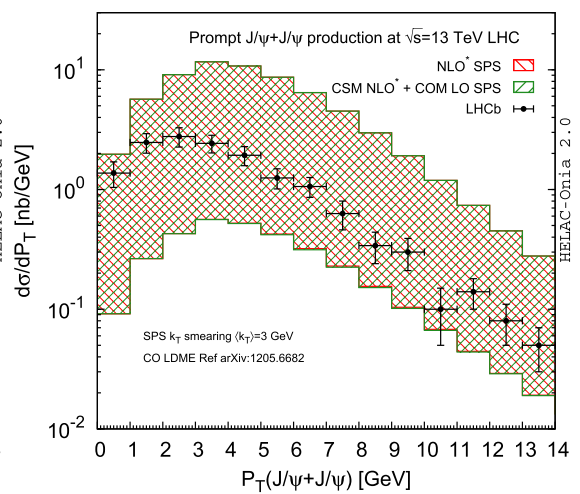

(f) Set 6

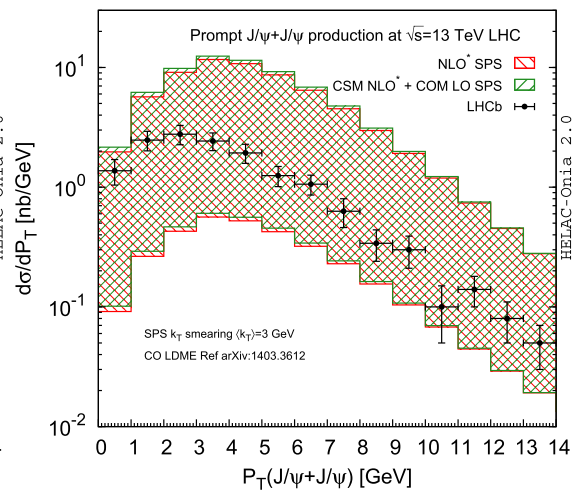

(i) Set 9

Fig. $14 P_{T}(J / \psi+J / \psi)$ distributions via SPS NLO ${ }^{\star}$ CS and LO CO in the LHCb acceptance at $\sqrt{s}=13 \mathrm{TeV}$ [23] 


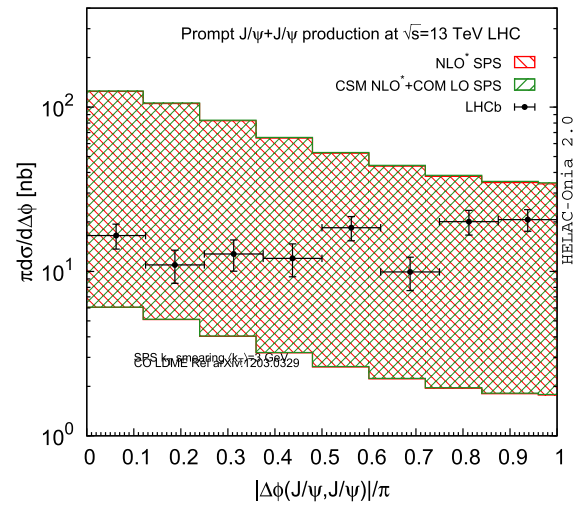

(a) Set 1

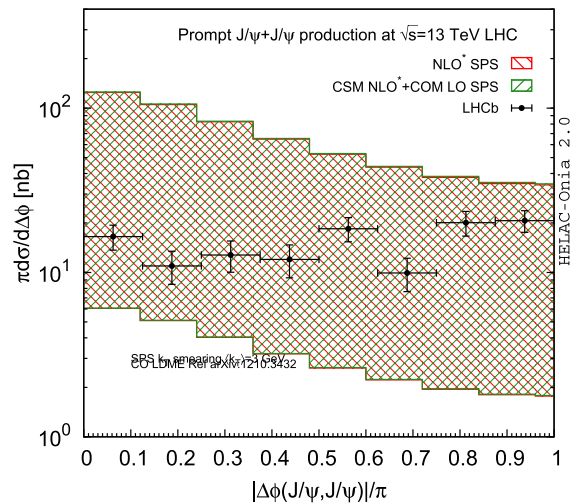

(d) Set 4

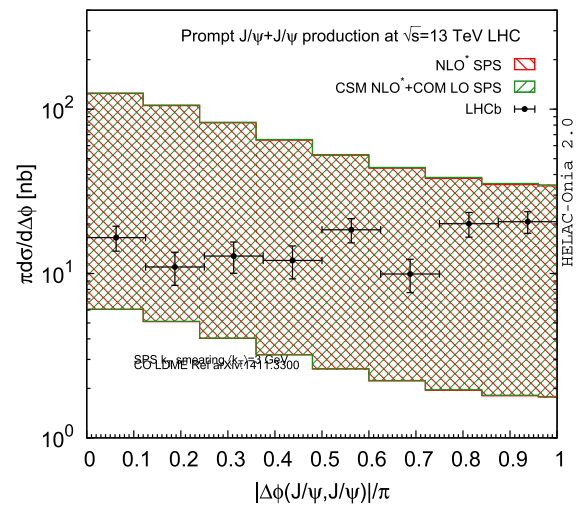

(g) Set 7

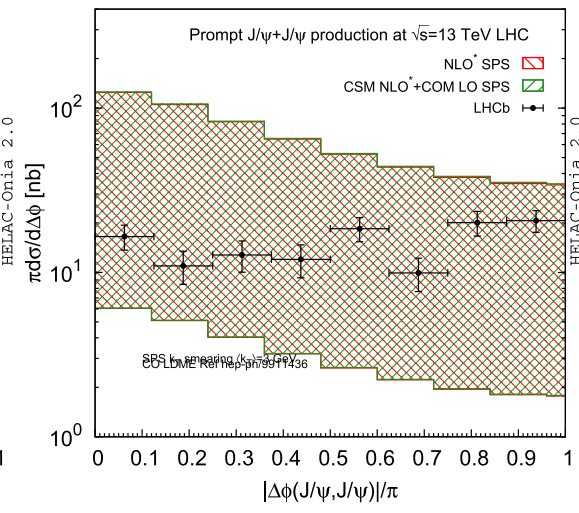

(b) Set 2

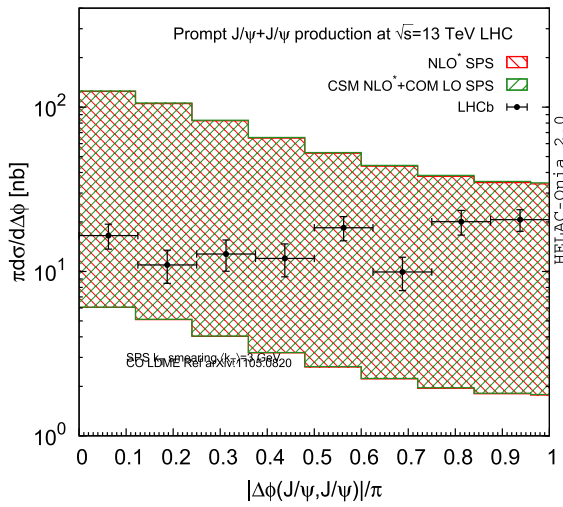

(e) Set 5

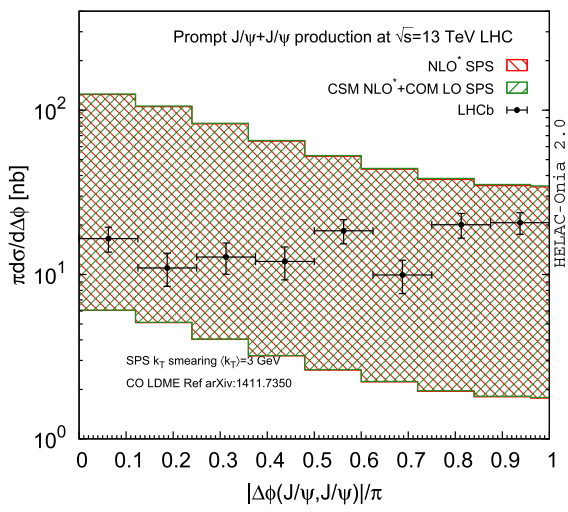

(h) Set 8

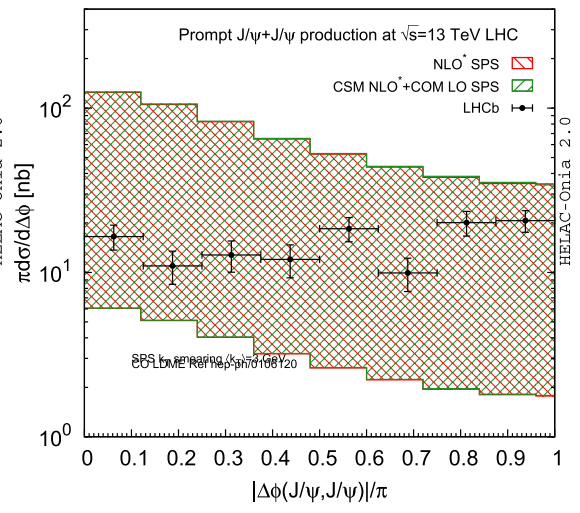

(c) Set 3

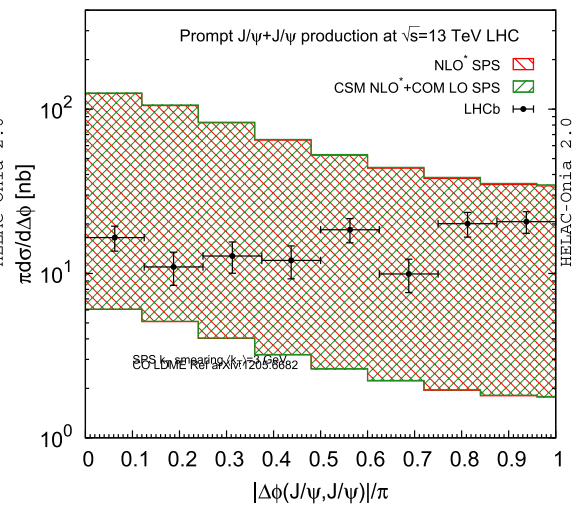

(f) Set 6

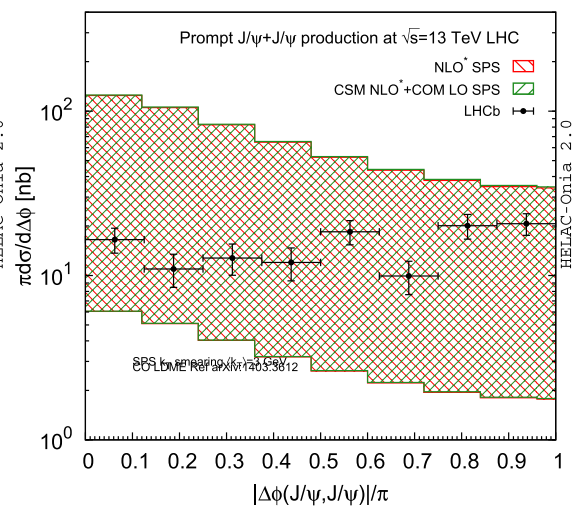

(i) Set 9

Fig. $15 \Delta \phi(J / \psi, J / \psi)$ distributions via SPS NLO ${ }^{\star}$ CS and LO CO in the LHCb acceptance at $\sqrt{s}=13 \mathrm{TeV}$ [23] 


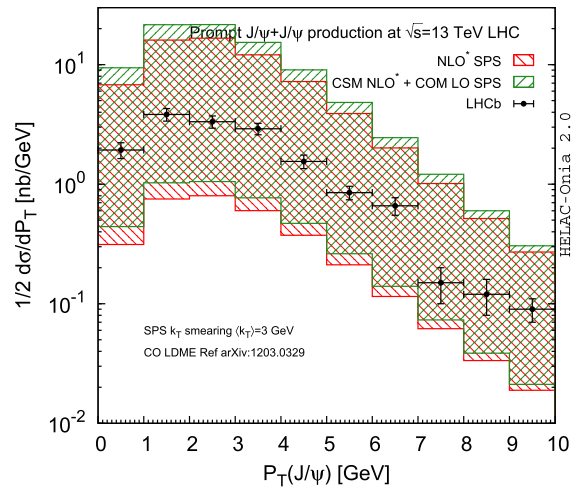

(a) Set 1



(d) Set 4

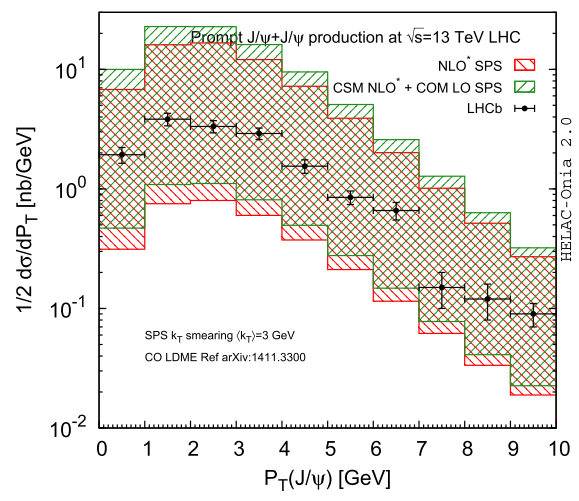

(g) Set 7

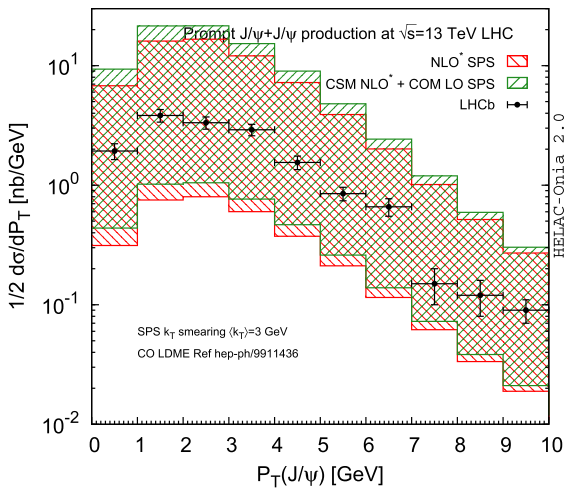

(b) Set 2



(e) Set 5

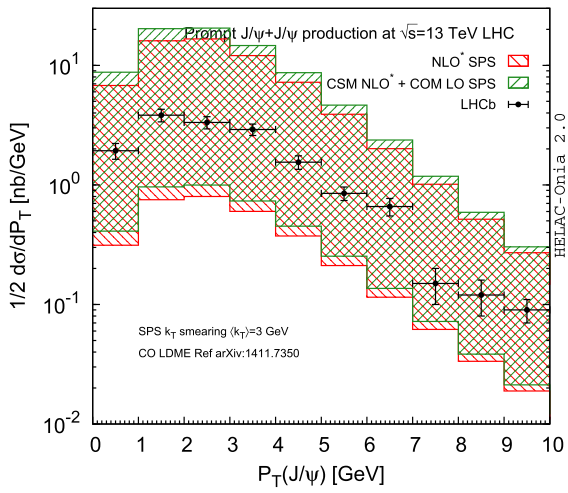

(h) Set 8

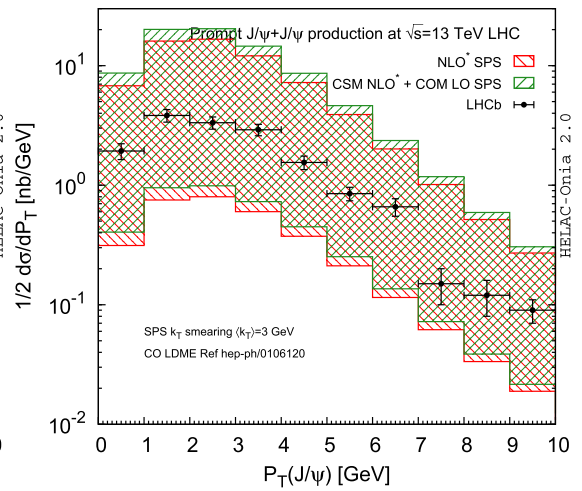

(c) Set 3

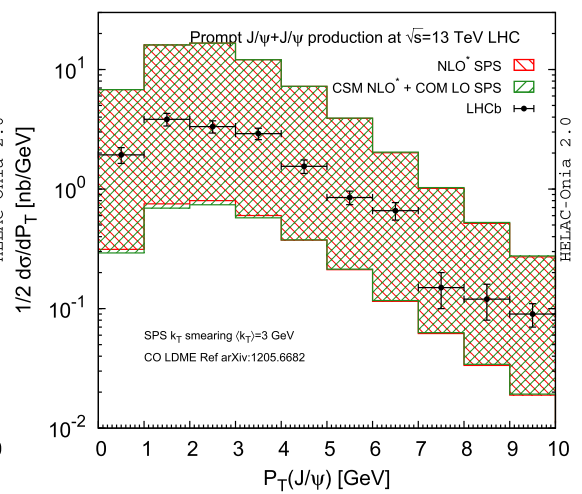

(f) Set 6

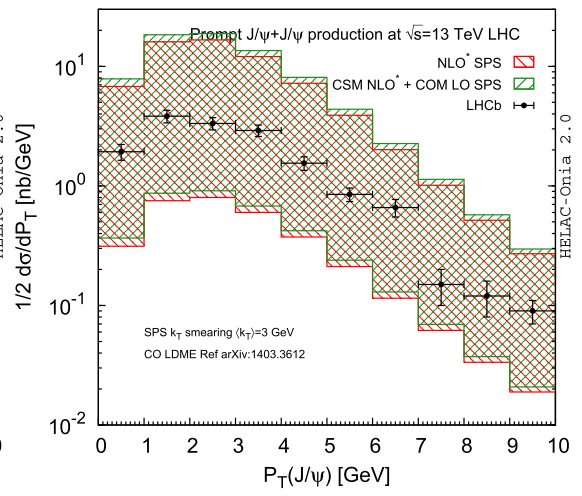

(i) Set 9

Fig. $16 P_{T}(J / \psi)$ distributions via SPS NLO ${ }^{\star}$ CS and LO CO in the LHCb acceptance at $\sqrt{s}=13 \mathrm{TeV}$ [23] 


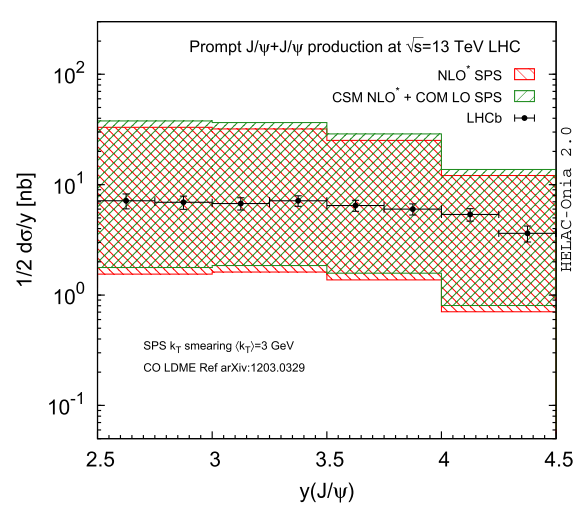

(a) Set 1

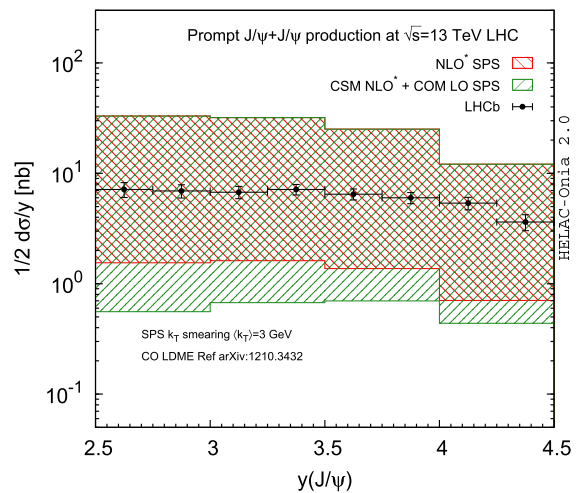

(d) Set 4

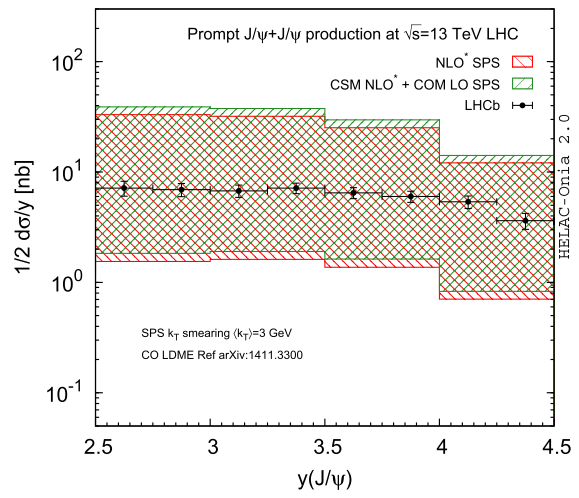

(g) Set 7

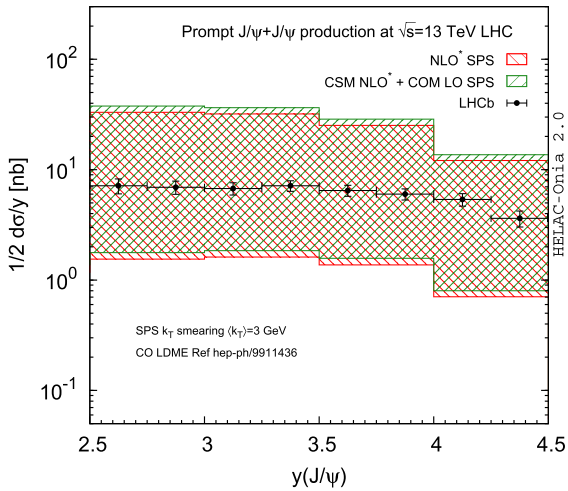

(b) Set 2

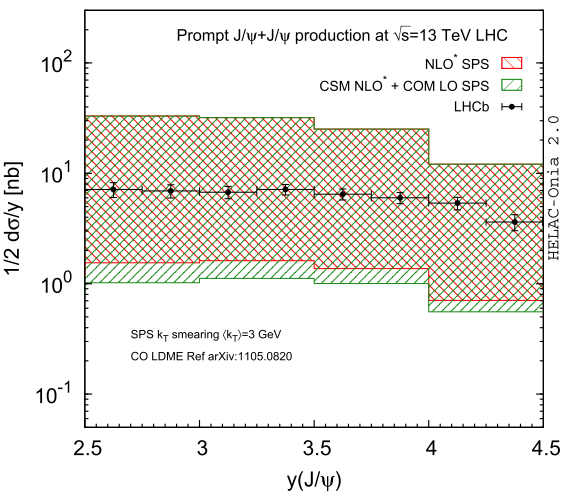

(e) Set 5

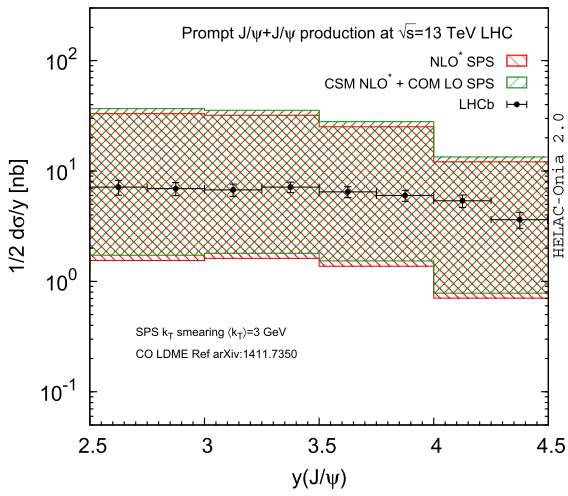

(h) Set 8

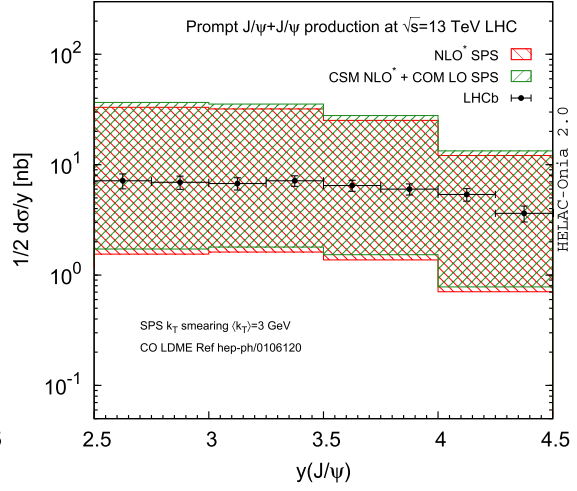

(c) Set 3

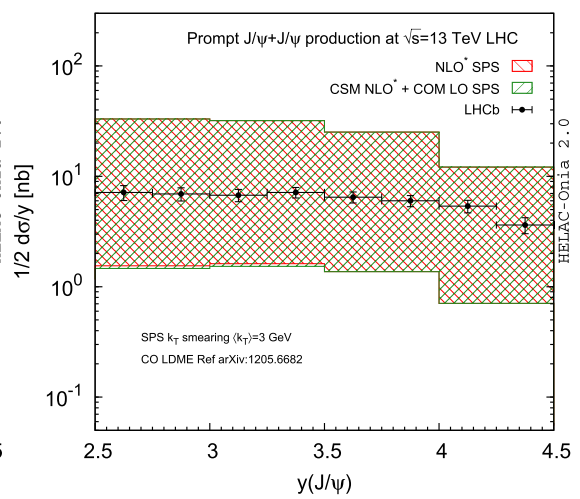

(f) Set 6

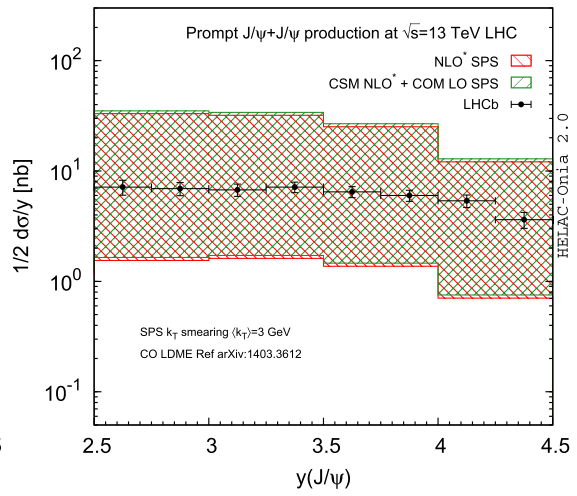

(i) Set 9

Fig. $17 y(J / \psi)$ distributions via SPS NLO ${ }^{\star}$ CS and LO CO in the LHCb acceptance at $\sqrt{s}=13 \mathrm{TeV}$ [23] 


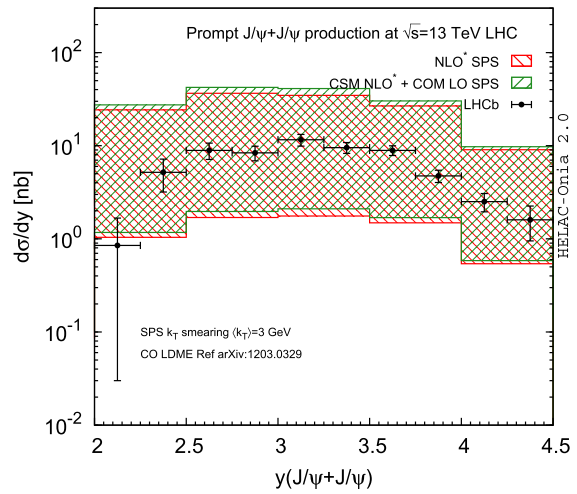

(a) Set 1

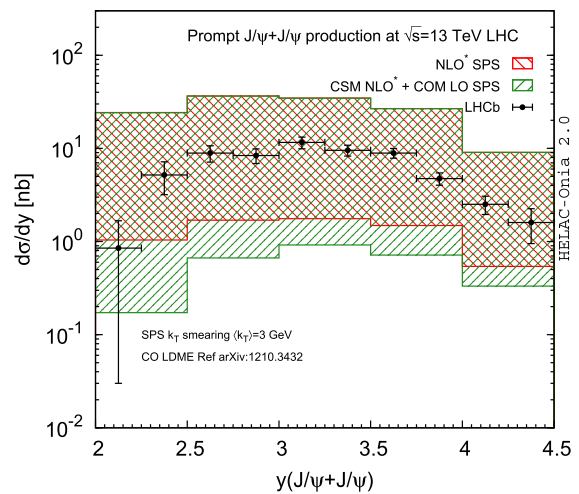

(d) Set 4

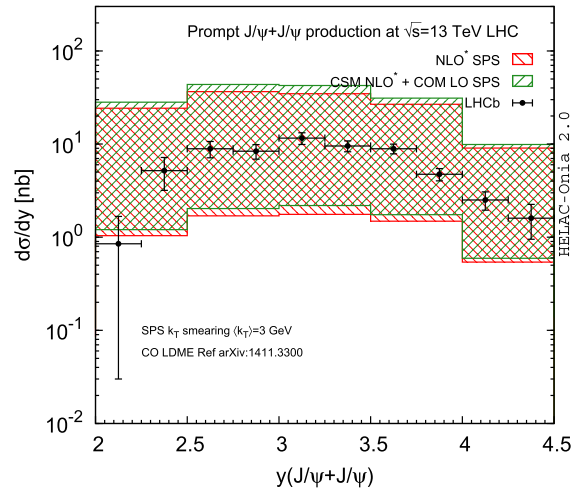

(g) Set 7



(b) Set 2

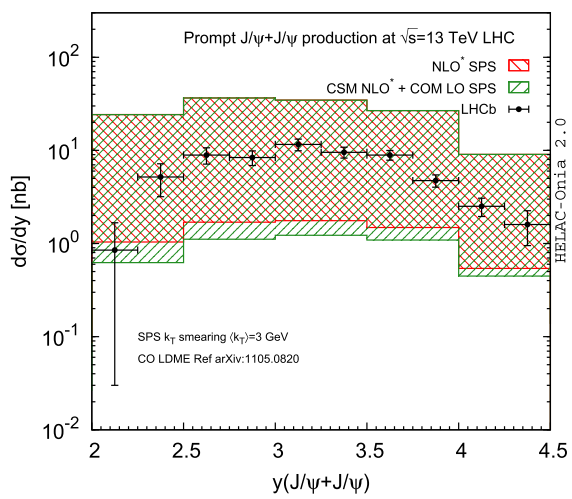

(e) Set 5



(h) Set 8

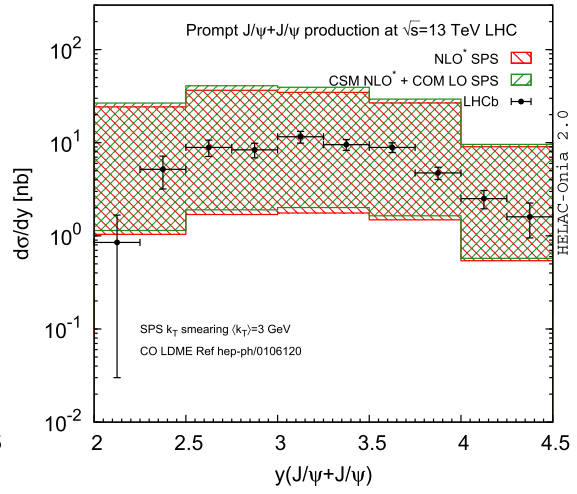

(c) Set 3

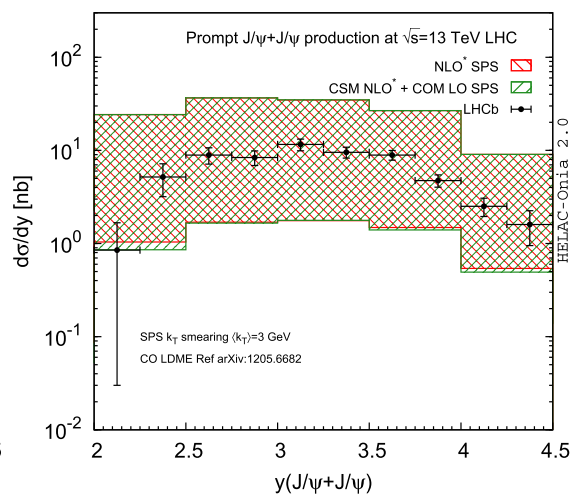

(f) Set 6

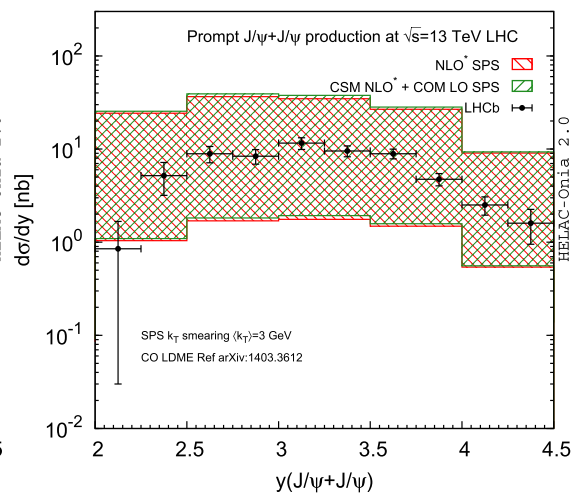

(i) Set 9

Fig. $18 y(J / \psi+J / \psi)$ distributions via SPS NLO ${ }^{\star}$ CS and LO CO in the LHCb acceptance at $\sqrt{s}=13 \mathrm{TeV}$ [23] 




(a) Set 1



(d) Set 4

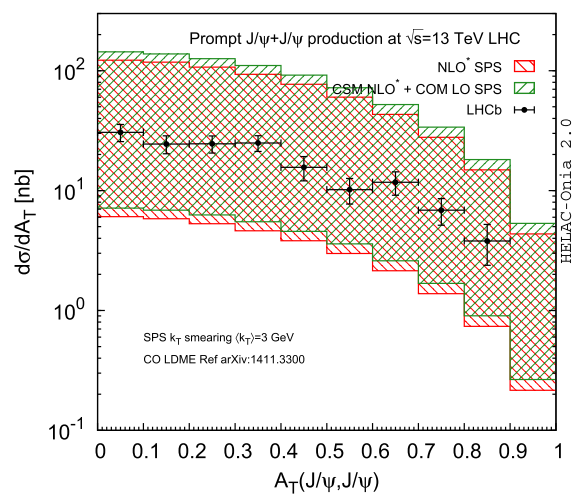

(g) Set 7

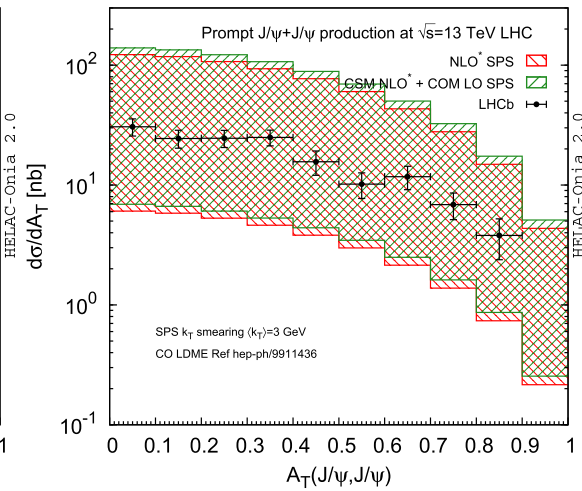

(b) Set 2

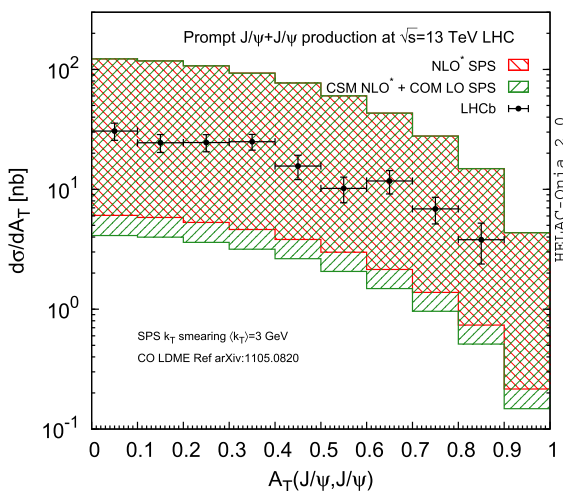

(e) Set 5

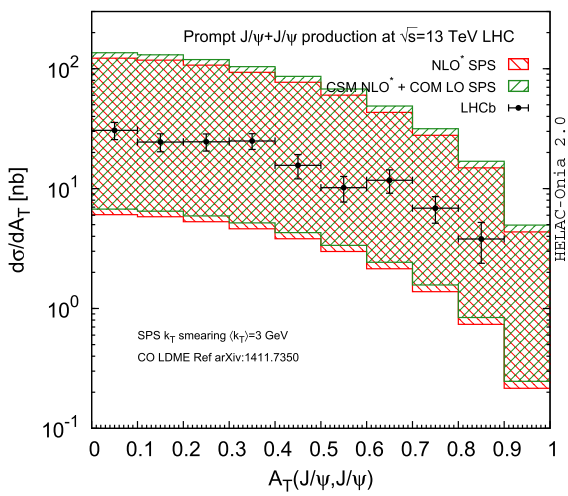

(h) Set 8

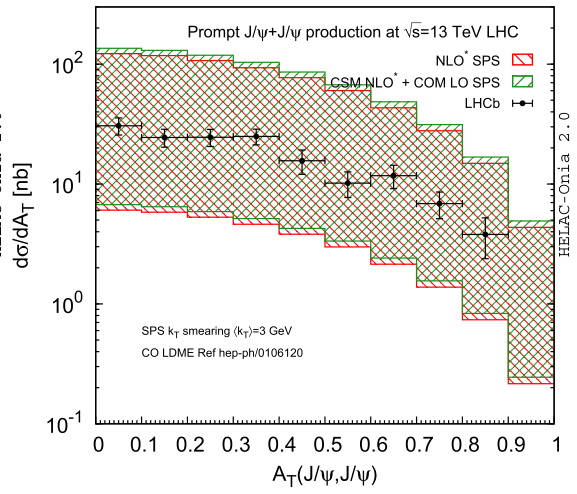

(c) Set 3

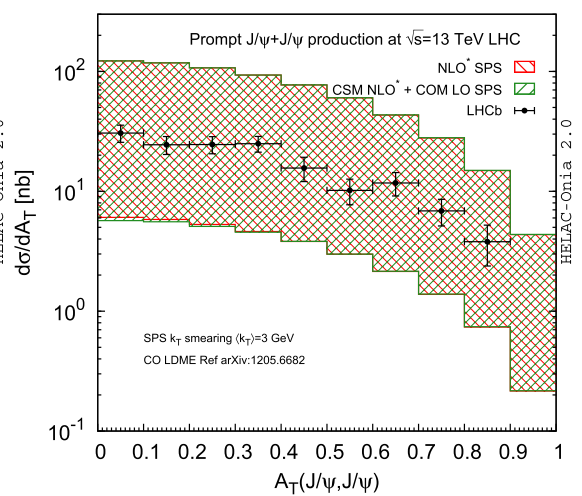

(f) Set 6

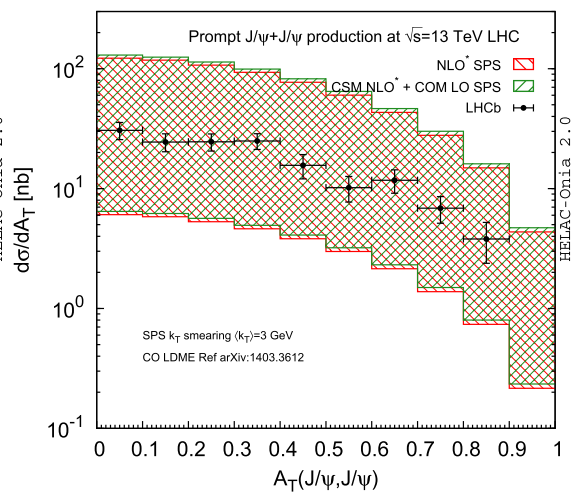

(i) Set 9

Fig. $19 A_{T}(J / \psi, J / \psi)$ distributions via SPS NLO ${ }^{\star}$ CS and LO CO in the LHCb acceptance at $\sqrt{s}=13 \mathrm{TeV}$ [23] 


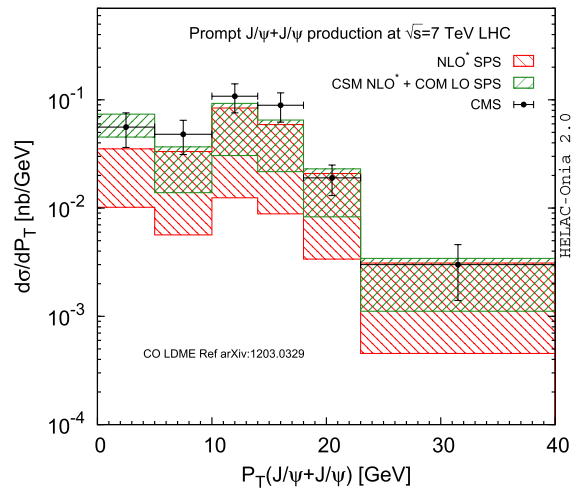

(a) Set 1

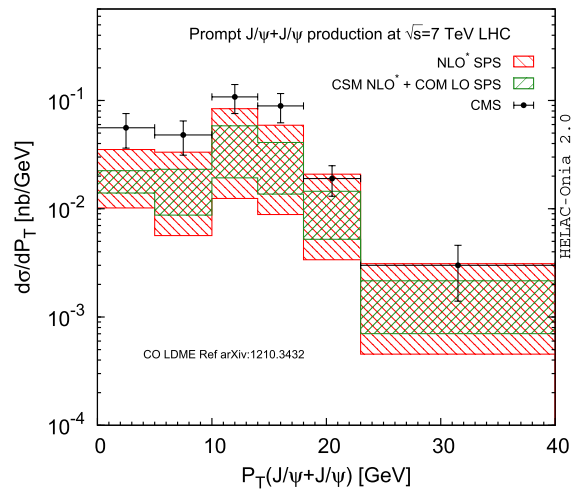

(d) Set 4

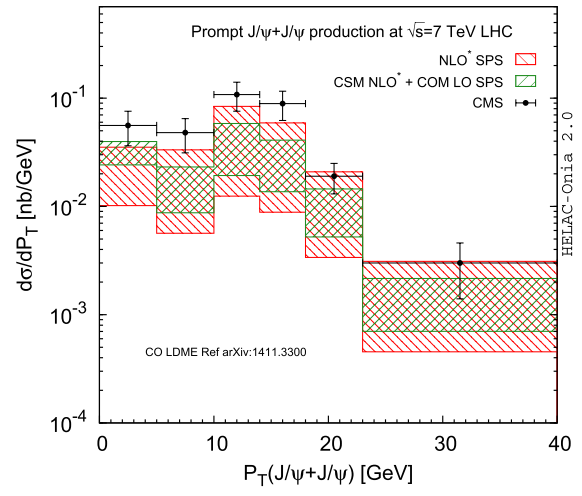

(g) Set 7

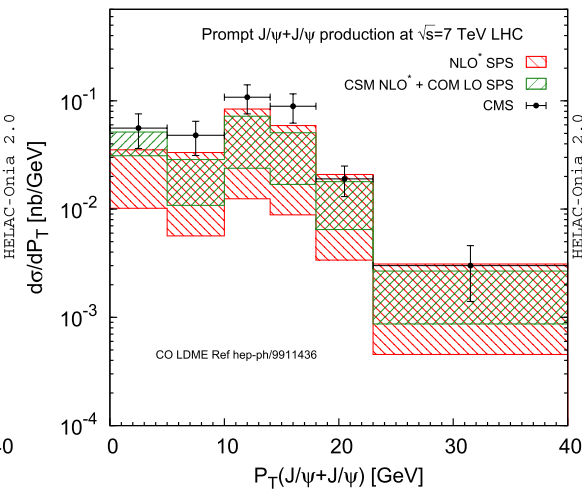

(b) Set 2



(e) Set 5

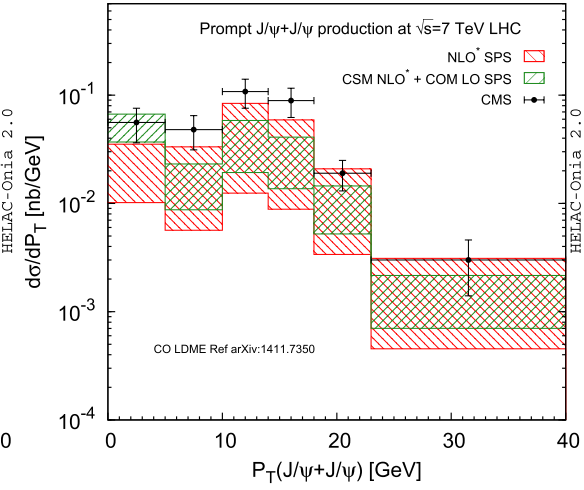

(h) Set 8

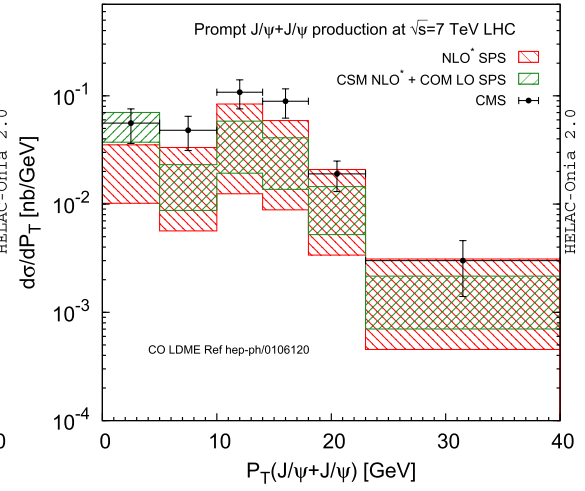

(c) Set 3

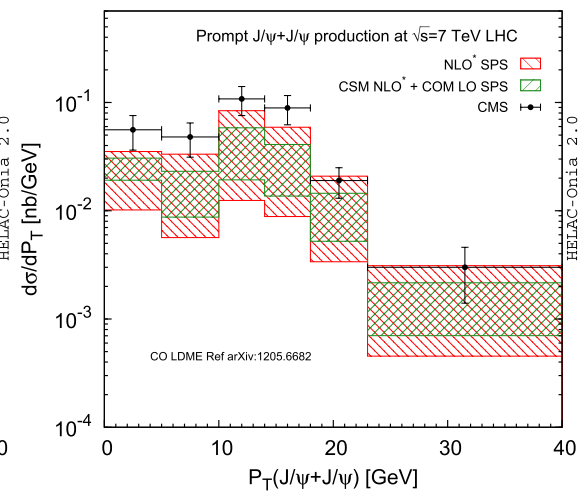

(f) Set 6

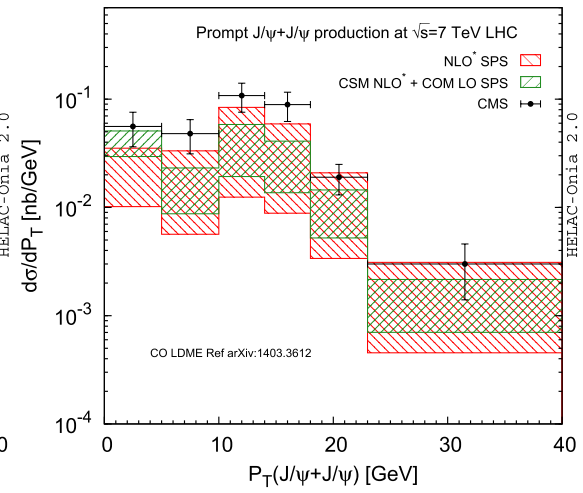

(i) Set 9

Fig. $20 P_{T}(J / \psi+J / \psi)$ distributions via SPS NLO^ CS and LO CO in the CMS acceptance at $\sqrt{s}=7 \mathrm{TeV}$ [21] 


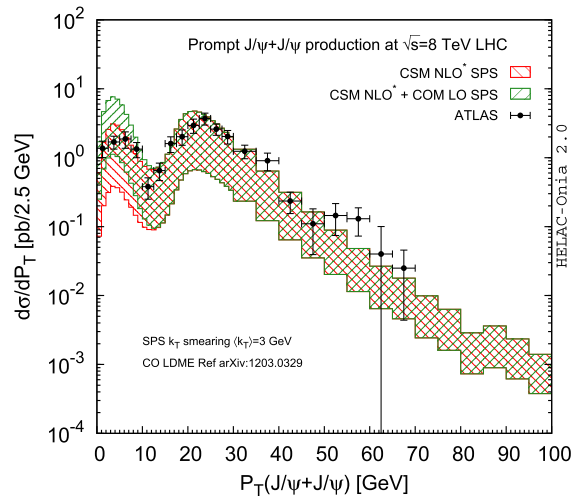

(a) Set 1

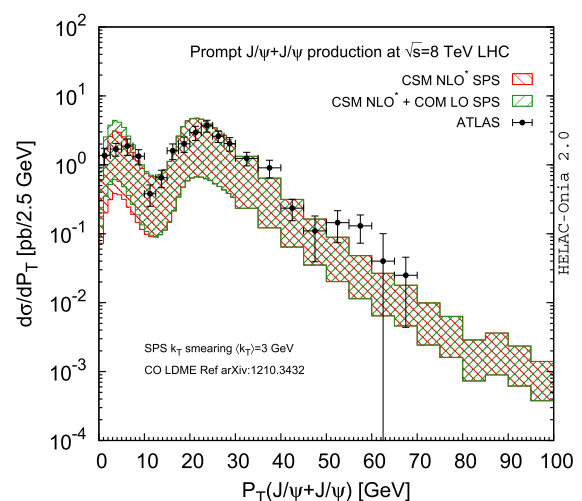

(d) Set 4

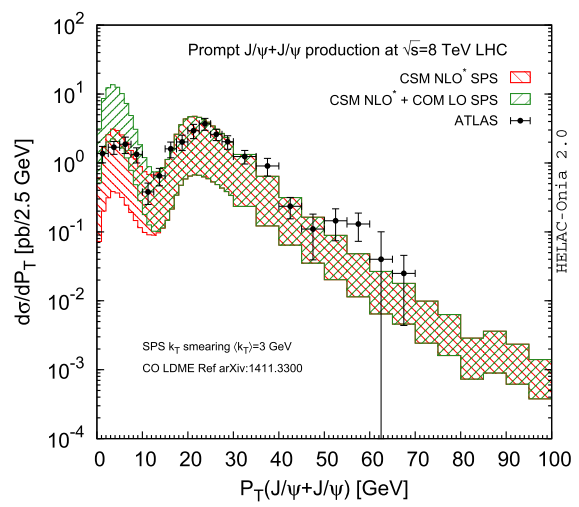

(g) Set 7

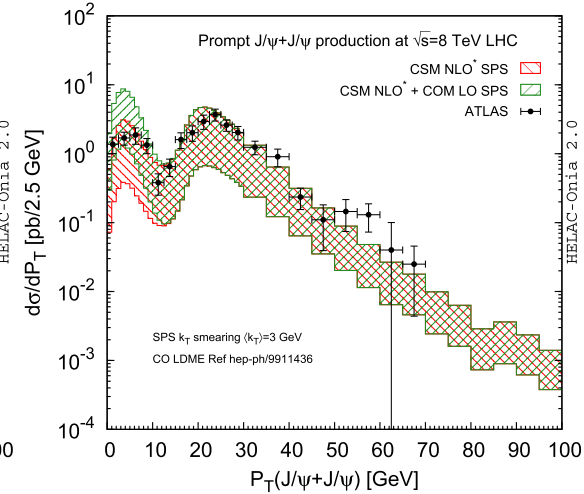

(b) Set 2

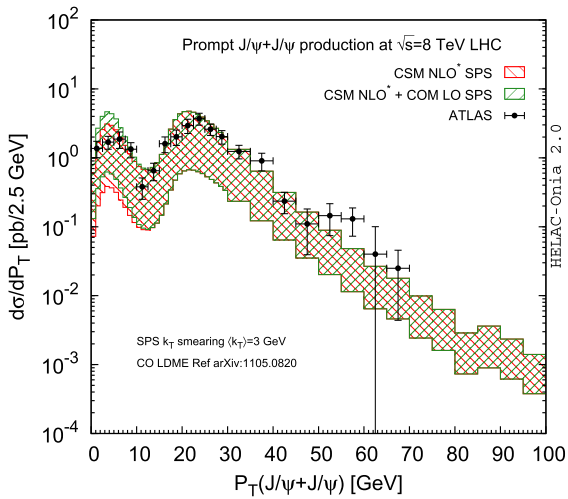

(e) Set 5

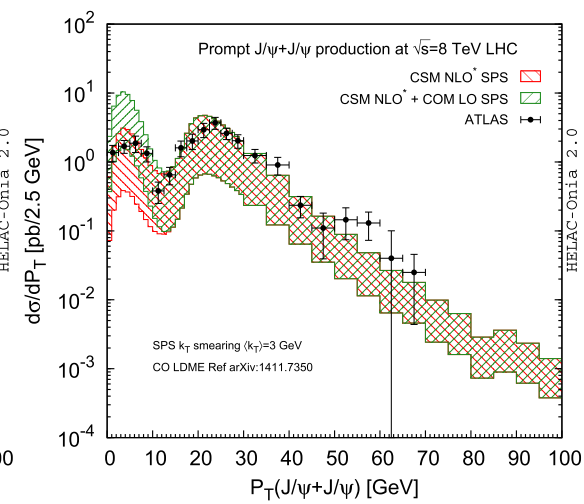

(h) Set 8

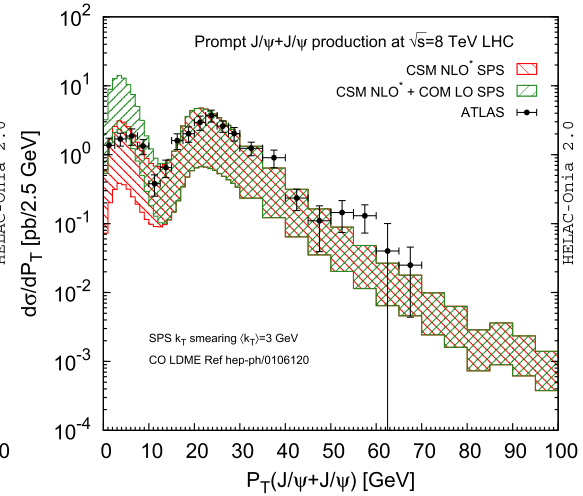

(c) Set 3

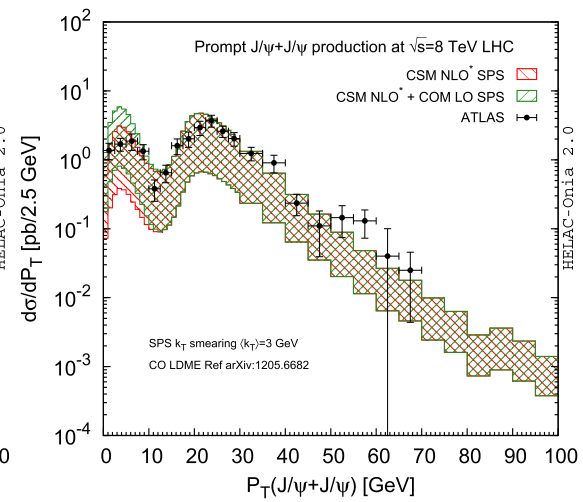

(f) Set 6



(i) Set 9

Fig. $21 P_{T}(J / \psi+J / \psi)$ distributions via SPS NLO ${ }^{\star}$ CS and LO CO in the ATLAS acceptance at $\sqrt{s}=8 \mathrm{TeV}$ [22] 


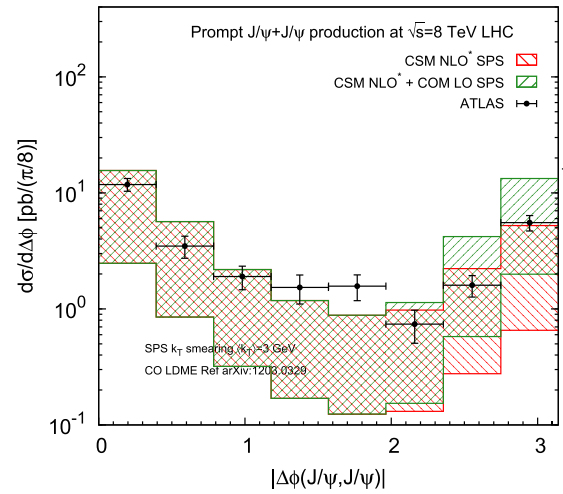

(a) Set 1

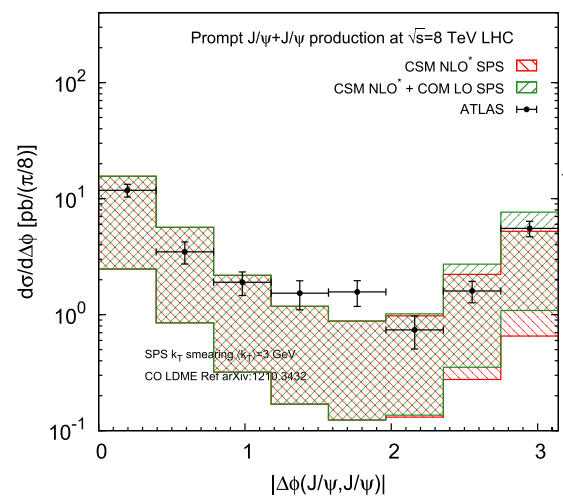

(d) Set 4

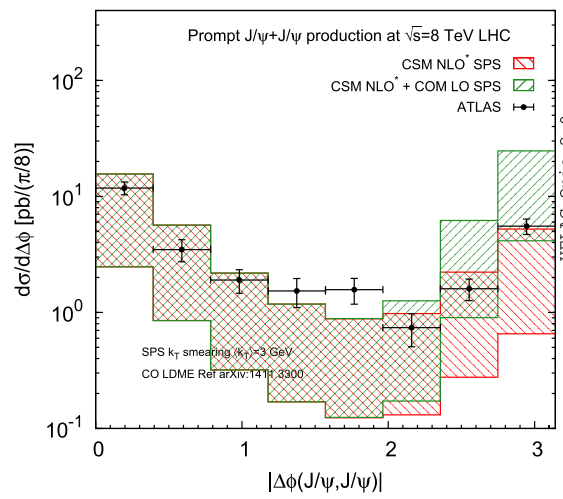

(g) Set 7

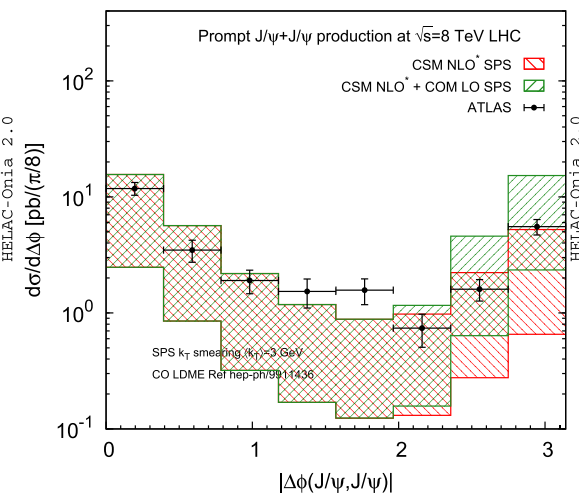

(b) Set 2

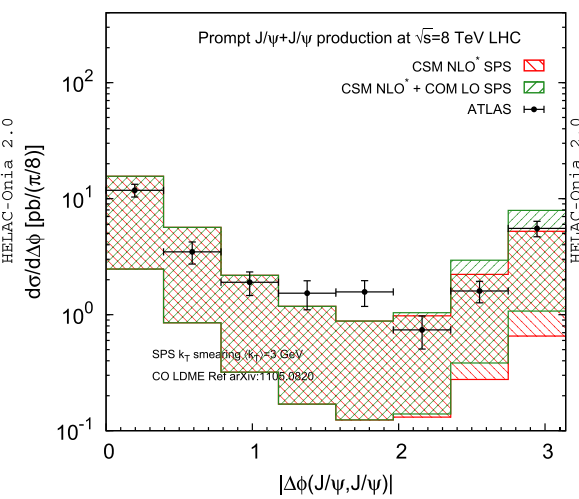

(e) Set 5

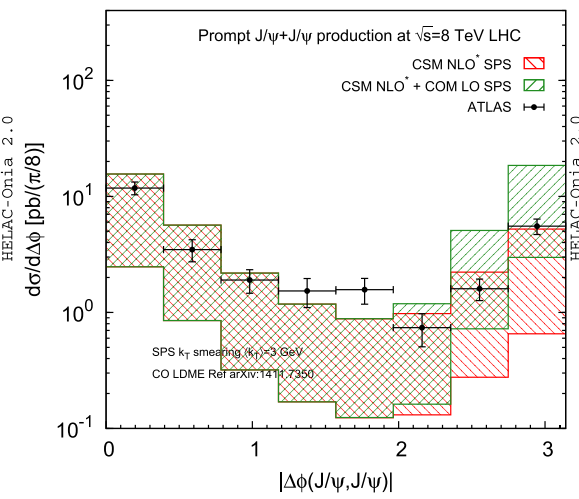

(h) Set 8

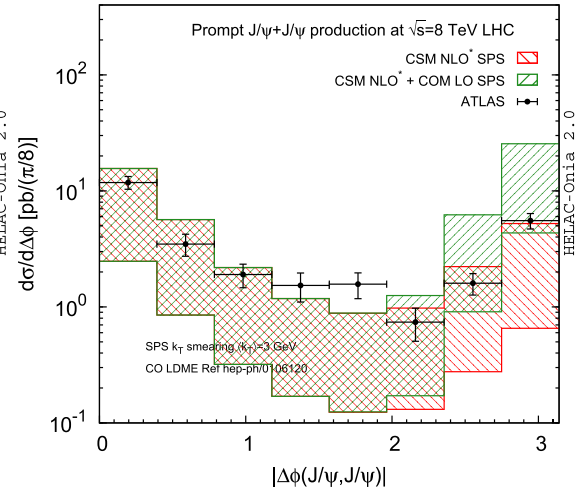

(c) Set 3



(f) Set 6

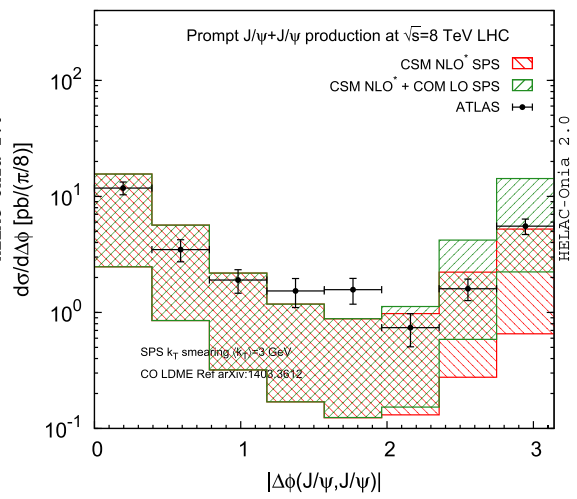

(i) Set 9

Fig. $22 \Delta \phi(J / \psi, J / \psi)$ distributions via SPS NLO ${ }^{\star}$ CS and LO CO in the ATLAS acceptance at $\sqrt{s}=8 \mathrm{TeV}$ acceptance [22]

\section{References}

1. B. Blok, Yu. Dokshitser, L. Frankfurt, M. Strikman, pQCD physics of multiparton interactions. Eur. Phys. J. C 72, 1963 (2012). arXiv:1106.5533 [hep-ph]

2. M. Diehl, D. Ostermeier, A. Schafer, Elements of a theory for multiparton interactions in QCD. JHEP 03, 089 (2012). arXiv: 1111.0910 [hep-ph]. [Erratum: JHEP03,001(2016)]

3. M. Diehl, A. Schafer, Theoretical considerations on multiparton interactions in QCD. Phys. Lett. B 698, 389-402 (2011). arXiv:1102.3081 [hep-ph]

4. J.R. Gaunt, W.J. Stirling, Double parton scattering singularity in one-loop integrals. JHEP 06, 048 (2011). arXiv:1103.1888 [hep$\mathrm{ph}]$
5. A.V. Manohar, W.J. Waalewijn, What is double parton scattering? Phys. Lett. B 713, 196-201 (2012). arXiv:1202.5034 [hep-ph]

6. J.R. Gaunt, Single perturbative splitting diagrams in double parton scattering. JHEP 01, 042 (2013). arXiv:1207.0480 [hep-ph]

7. B. Blok, Yu. Dokshitzer, L. Frankfurt, M. Strikman, Perturbative QCD correlations in multi-parton collisions. Eur. Phys. J. C 74, 2926 (2014). arXiv:1306.3763 [hep-ph]

8. M. Diehl, T. Kasemets, S. Keane, Correlations in double parton distributions: effects of evolution. JHEP 05, 118 (2014). arXiv:1401.1233 [hep-ph]

9. M. Diehl, J.R. Gaunt, D. Ostermeier, P. Ploessl, A. Schaefer, Cancellation of Glauber gluon exchange in the double Drell-Yan process. JHEP 01, 076 (2016). arXiv:1510.08696 [hep-ph] 
10. M. Rinaldi, S. Scopetta, M.C. Traini, V. Vento, Correlations in double parton distributions: perturbative and non-perturbative effects. JHEP 10, 063 (2016). arXiv:1608.02521 [hep-ph]

11. M.G.A. Buffing, M. Diehl, T. Kasemets, Transverse momentum in double parton scattering: factorisation, evolution and matching. JHEP 01, 044 (2018). arXiv:1708.03528 [hep-ph]

12. M. Diehl, J.R. Gaunt, K. Schoenwald, Double hard scattering without double counting. JHEP 06, 083 (2017). arXiv:1702.06486 [hep$\mathrm{ph}]$

13. A. Vladimirov, Structure of rapidity divergences in multi-parton scattering soft factors. JHEP 04, 045 (2018). arXiv:1707.07606 [hep-ph]

14. M. Diehl, R. Nagar, Factorisation of soft gluons in multiparton scattering. JHEP 04, 124 (2019). arXiv:1812.09509 [hep-ph]

15. J.R. Gaunt, T. Kasemets, Transverse momentum dependence in double parton scattering. Adv. High Energy Phys. 2019, 3797394 (2019). arXiv:1812.09099 [hep-ph]

16. M. Rinaldi, F.A. Ceccopieri, Double parton scattering and the proton transverse structure at the LHC. arXiv:1812.04286 [hep-ph]

17. M. Rinaldi, F.A. Ceccopieri, Hadronic structure from double parton scattering. Phys. Rev. D 97(7), 071501 (2018). arXiv:1801.04760 [hep-ph]

18. J.-P. Lansberg, New observables in inclusive production of quarkonia. arXiv:1903.09185 [hep-ph]

19. LHCb Collaboration, R. Aaij et al., Observation of $J / \psi$ pair production in $p p$ collisions at $\sqrt{s}=7 T e V$. Phys. Lett. B 707, 52-59 (2012). arXiv:1109.0963 [hep-ex]

20. D0 Collaboration, V.M. Abazov et al., Observation and studies of double $J / \psi$ production at the tevatron. Phys. Rev. D 90(11), 111101 (2014). arXiv:1406.2380 [hep-ex]

21. C.M.S. Collaboration, V. Khachatryan et al., Measurement of prompt $J / \psi$ pair production in pp collisions at $\sqrt{s}=7 \mathrm{TeV}$. JHEP 09, 094 (2014). arXiv:1406.0484 [hep-ex]

22. ATLAS Collaboration, M. Aaboud et al., Measurement of the prompt $\mathrm{J} / \psi$ pair production cross-section in pp collisions at $\sqrt{s}=8$ TeV with the ATLAS detector. Eur. Phys. J. C 77(2), 76 (2017). arXiv:1612.02950 [hep-ex]

23. LHCb Collaboration, R. Aaij et al., Measurement of the $\mathrm{J} / \psi$ pair production cross-section in pp collisions at $\sqrt{s}=13 \mathrm{TeV}$. JHEP 06 047 (2017). arXiv:1612.07451 [hep-ex]. [Erratum: JHEP 10, 068 (2017)]

24. D0 Collaboration, V.M. Abazov et al., Evidence for simultaneous production of $J / \psi$ and $\Upsilon$ mesons. Phys. Rev. Lett. 116(8), 082002 (2016). arXiv:1511.02428 [hep-ex]

25. C.M.S. Collaboration, V. Khachatryan et al., Observation of $\Upsilon(1 S)$ pair production in proton-proton collisions at $\sqrt{s}=8 \mathrm{TeV}$. JHEP 05, 013 (2017). arXiv:1610.07095 [hep-ex]

26. ATLAS Collaboration, G. Aad et al., Measurement of the production cross section of prompt $J / \psi$ mesons in association with a $W^{ \pm}$ boson in $p p$ collisions at $\sqrt{s}=7 \mathrm{TeV}$ with the ATLAS detector. JHEP 04, 172 (2014). arXiv:1401.2831 [hep-ex]

27. ATLAS Collaboration, G. Aad et al., Observation and measurements of the production of prompt and non-prompt $J / \psi$ mesons in association with a $Z$ boson in $p p$ collisions at $\sqrt{s}=8 \mathrm{TeV}$ with the ATLAS detector. Eur. Phys. J. C 75(5), 229 (2015). arXiv: 1412.6428 [hep-ex]

28. LHCb Collaboration, R. Aaij et al., Observation of double charm production involving open charm in pp collisions at $\sqrt{s}=7$ TeV. JHEP 06, 141 (2012). arXiv:1205.0975 [hep-ex]. [Addendum: JHEP 03, 108 (2014)]

29. LHCb Collaboration, R. Aaij et al., Production of associated $Y$ and open charm hadrons in pp collisions at $\sqrt{s}=7$ and $8 \mathrm{TeV}$ via double parton scattering. JHEP 07, 052 (2016). arXiv: 1510.05949 [hep-ex]
30. R. Li, Y.-J. Zhang, K.-T. Chao, Pair production of heavy quarkonium and $\mathrm{B}(\mathrm{c})(*)$ mesons at hadron colliders. Phys. Rev. D 80, 014020 (2009). arXiv:0903.2250 [hep-ph]

31. C.-F. Qiao, L.-P. Sun, P. Sun, Testing charmonium production mechamism via polarized J/psi pair production at the LHC. J. Phys. G 37, 075019 (2010). arXiv:0903.0954 [hep-ph]

32. P. Ko, C. Yu, J. Lee, Inclusive double-quarkonium production at the Large Hadron Collider. JHEP 01, 070 (2011). arXiv:1007.3095 [hep-ph]

33. C.H. Kom, A. Kulesza, W.J. Stirling, Pair production of J/psi as a probe of double parton scattering at LHCb. Phys. Rev. Lett. 107, 082002 (2011). arXiv:1105.4186 [hep-ph]

34. A.V. Berezhnoy, A.K. Likhoded, A.V. Luchinsky, A.A. Novoselov, Double J/psi-meson Production at LHC and 4c-tetraquark state. Phys. Rev. D 84, 094023 (2011). arXiv:1101.5881 [hep-ph]

35. J.-P. Lansberg, H.-S. Shao, Production of $J / \psi+\eta_{c}$ versus $J / \psi+$ $J / \psi$ at the LHC: importance of real $\alpha_{s}^{5}$ corrections. Phys. Rev. Lett. 111, 122001 (2013). arXiv:1308.0474 [hep-ph]

36. Y.-J. Li, G.-Z. Xu, K.-Y. Liu, Y.-J. Zhang, Relativistic correction to J/psi and upsilon pair production. JHEP 07, 051 (2013). arXiv:1303.1383 [hep-ph]

37. J.-P. Lansberg, H.-S. Shao, J/ $\psi$-pair production at large momenta: indications for double parton scatterings and large $\alpha_{s}^{5}$ contributions. Phys. Lett. B 751, 479-486 (2015). arXiv:1410.8822 [hep-ph]

38. L.-P. Sun, H. Han, K.-T. Chao, Impact of $J / \psi$ pair production at the LHC and predictions in nonrelativistic QCD. Phys. Rev. D 94(7), 074033 (2016). arXiv:1404.4042 [hep-ph]

39. J.-P. Lansberg, H.-S. Shao, Double-quarkonium production at a fixed-target experiment at the LHC (AFTER@LHC). Nucl. Phys. B 900, 273-294 (2015). arXiv:1504.06531 [hep-ph]

40. Z.-G. He, B.A. Kniehl, Complete nonrelativistic-QCD prediction for prompt double J/hadroproduction. Phys. Rev. Lett. 115(2), 022002 (2015). arXiv:1609.02786 [hep-ph]

41. S.P. Baranov, A.H. Rezaeian, Prompt double $J / \psi$ production in proton-proton collisions at the LHC. Phys. Rev. D 93(11), 114011 (2016). arXiv:1511.04089 [hep-ph]

42. H.-S. Shao, Y.-J. Zhang, Complete study of hadroproduction of a $\Upsilon$ meson associated with a prompt $J / \psi$. Phys. Rev. Lett. 117(6), 062001 (2016). arXiv:1605.03061 [hep-ph]

43. J.-P. Lansberg, H.-S. Shao, Associated production of a quarkonium and a $\mathrm{Z}$ boson at one loop in a quark-hadron-duality approach. JHEP 10, 153 (2016). arXiv:1608.03198 [hep-ph]

44. J.-P. Lansberg, H.-S. Shao, Phenomenological analysis of associated production of $Z^{0}+b$ in the $b \rightarrow J / \psi X$ decay channel at the LHC. Nucl. Phys. B 916, 132-142 (2017). arXiv:1611.09303 [hep-ph]

45. A.K. Likhoded, A.V. Luchinsky, S.V. Poslavsky, Production of $J / \psi+\chi_{c}$ and $J / \psi+J / \psi$ with real gluon emission at LHC. Phys. Rev. D 94(5), 054017 (2016). arXiv:1606.06767 [hep-ph]

46. C. Borschensky, A. Kulesza, Double parton scattering in pair production of $J / \psi$ mesons at the LHC revisited. Phys. Rev. D 95(3), 034029 (2017). arXiv:1610.00666 [hep-ph]

47. J.-P. Lansberg, H.-S. Shao, N. Yamanaka, Indication for double parton scatterings in $W+$ prompt $J / \psi$ production at the LHC. Phys. Lett. B 781, 485-491 (2018). arXiv:1707.04350 [hep-ph]

48. J.-P. Lansberg, C. Pisano, F. Scarpa, M. Schlegel, Pinning down the linearly-polarised gluons inside unpolarised protons using quarkonium-pair production at the LHC. Phys. Lett. B 784, 217222 (2018). arXiv: 1710.01684 [hep-ph]

49. A. Cisek, W. Schaefer, A. Szczurek, Production of $\chi_{c}$ pairs with large rapidity separation in $k_{T}$ factorization. Phys. Rev. D 97(11), 114018 (2018). arXiv:1711.07366 [hep-ph]

50. A. Gridin, S. Groote, A. Guskov, S. Koshkarev, Feasibility study for the search of intrinsic charm at the COMPASS experiment and at the STAR fixed-target program. arXiv:1901.01712 [hep-ph] 
51. Z.-G. He, B.A. Kniehl, M.A. Nefedov, V.A. Saleev, Double prompt $J / \psi$ hadroproduction in the parton reggeization approach with high-energy resummation. arXiv:1906.08979 [hep-ph]

52. H.-S. Shao, Y.-J. Zhang, Triple prompt $J / \psi$ hadroproduction as a hard probe of multiple-parton scatterings. Phys. Rev. Lett. 122(19), 192002 (2019). arXiv:1902.04949 [hep-ph]

53. G.T. Bodwin, E. Braaten, G.P. Lepage, Rigorous QCD analysis of inclusive annihilation and production of heavy quarkonium. Phys. Rev. D 51, 1125-1171 (1995). arXiv:hep-ph/9407339 [hep-ph]. [Erratum: Phys. Rev. D 55, 5853 (1997)]

54. K. Sridhar, A.D. Martin, W.J. Stirling, $J / \psi$ production at the tevatron and HERA: the effect of $k_{T}$ smearing. Phys. Lett. B 438, 211-216 (1998). arXiv:hep-ph/9806253 [hep-ph]

55. H.-S. Shao, HELAC-Onia: an automatic matrix element generator for heavy quarkonium physics. Comput. Phys. Commun. 184, 2562-2570 (2013). arXiv:1212.5293 [hep-ph]

56. H.-S. Shao, HELAC-Onia 2.0: an upgraded matrix-element and event generator for heavy quarkonium physics. Comput. Phys. Commun. 198, 238-259 (2016). arXiv:1507.03435 [hep-ph]

57. J. Pumplin, D.R. Stump, J. Huston, H.L. Lai, P.M. Nadolsky, W.K. Tung, New generation of parton distributions with uncertainties from global QCD analysis. JHEP 07, 012 (2002). arXiv:hep-ph/0201195 [hep-ph]

58. NNPDF Collaboration, R.D. Ball et al., Parton distributions for the LHC Run II. JHEP 04, 040 (2015). arXiv:1410.8849 [hep-ph]

59. E.J. Eichten, C. Quigg, Quarkonium wave functions at the origin. Phys. Rev. D 52, 1726-1728 (1995). arXiv:hep-ph/9503356 [hep$\mathrm{ph}]$

60. W. Buchmuller, S.H.H. Tye, Quarkonia and quantum chromodynamics. Phys. Rev. D 24, 132 (1981)

61. E. Braaten, B.A. Kniehl, J. Lee, Polarization of prompt $J / \psi$ at the Tevatron. Phys. Rev. D 62, 094005 (2000). arXiv:hep-ph/9911436 [hep-ph]

62. H.-S. Shao, Boosting perturbative QCD stability in quarkonium production. JHEP 01, 112 (2019). arXiv:1809.02369 [hep-ph]

63. Z.-G. He, B.A. Kniehl, X.-P. Wang, Breakdown of nonrelativistic QCD factorization in processes involving two quarkonia and its cure. Phys. Rev. Lett. 121(17), 172001 (2018). arXiv:1809.07993 [hep-ph]

64. R. Sharma, I. Vitev, High transverse momentum quarkonium production and dissociation in heavy ion collisions. Phys. Rev. C 87(4), 044905 (2013). arXiv:1203.0329 [hep-ph]

65. M. Kramer, Quarkonium production at high-energy colliders. Prog. Part. Nucl. Phys. 47, 141-201 (2001). arXiv:hep-ph/0106120 [hep$\mathrm{ph}]$

66. P. Sun, C.P. Yuan, F. Yuan, Heavy quarkonium production at low $\mathrm{Pt}$ in NRQCD with soft gluon resummation. Phys. Rev. D 88, 054008 (2013). arXiv:1210.3432 [hep-ph]

67. M. Butenschoen, B.A. Kniehl, World data of J/psi production consolidate NRQCD factorization at NLO. Phys. Rev. D 84, 051501 (2011). arXiv:1105.0820 [hep-ph]
68. B. Gong, L.-P. Wan, J.-X. Wang, H.-F. Zhang, Polarization for prompt J/psi and psi(2s) production at the tevatron and LHC. Phys. Rev. Lett. 110(4), 042002 (2013). arXiv:1205.6682 [hep-ph]

69. H.S. Shao, H. Han, Y.Q. Ma, C. Meng, Y.J. Zhang, K.T. Chao, Yields and polarizations of prompt $J / \psi$ and $\psi(2 S)$ production in hadronic collisions. JHEP 05, 103 (2015). arXiv:1411.3300 [hep$\mathrm{ph}]$

70. H. Han, Y.-Q. Ma, C. Meng, H.-S. Shao, K.-T. Chao, $\eta_{c}$ production at LHC and indications on the understanding of $J / \psi$ production. Phys. Rev. Lett. 114(9), 092005 (2015). arXiv:1411.7350 [hep-ph]

71. G.T. Bodwin, H.S. Chung, U.-R. Kim, J. Lee, Fragmentation contributions to $J / \psi$ production at the Tevatron and the LHC. Phys. Rev. Lett. 113(2), 022001 (2014). arXiv:1403.3612 [hep-ph]

72. S.J. Brodsky, J.-P. Lansberg, Heavy-quarkonium production in high energy proton-proton collisions at RHIC. Phys. Rev. D 81, 051502 (2010). arXiv:0908.0754 [hep-ph]

73. Y. Feng, J.-P. Lansberg, J.-X. Wang, Energy dependence of directquarkonium production in $p p$ collisions from fixed-target to LHC energies: complete one-loop analysis. Eur. Phys. J. C 75(7), 313 (2015). arXiv:1504.00317 [hep-ph]

74. R. Bain, L. Dai, A. Leibovich, Y. Makris, T. Mehen, NRQCD confronts LHCb data on quarkonium production within jets. Phys. Rev. Lett. 119(3), 032002 (2017). arXiv:1702.05525 [hep-ph]

75. R. Li, J.-X. Wang, Next-to-leading-order study of the associated production of $J / \psi+\gamma$ at the LHC. Phys. Rev. D 89(11), 114018 (2014). arXiv:1401.6918 [hep-ph]

76. LHCb Collaboration, R. Aaij et al., Measurement of $J / \psi$ polarization in $p p$ collisions at $\sqrt{s}=7 \mathrm{TeV}$. Eur. Phys. J. C 73(11), 2631 (2013). arXiv:1307.6379 [hep-ex]

77. Y.-Q. Ma, K. Wang, K.-T. Chao, $J / \psi\left(\psi^{\prime}\right)$ production at the Tevatron and LHC at $\mathcal{O}\left(\alpha_{s}^{4} v^{4}\right)$ in nonrelativistic QCD. Phys. Rev. Lett. 106, 042002 (2011). arXiv:1009.3655 [hep-ph]

78. K.-T. Chao, Y.-Q. Ma, H.-S. Shao, K. Wang, Y.-J. Zhang, $J / \psi$ polarization at hadron colliders in nonrelativistic QCD. Phys. Rev. Lett. 108, 242004 (2012). arXiv:1201.2675 [hep-ph]

79. CDF Collaboration, A. Abulencia et al., Polarization of $J / \psi$ and $\psi_{2 S}$ mesons produced in $p \bar{p}$ collisions at $\sqrt{s}=1.96-\mathrm{TeV}$. Phys. Rev. Lett. 99, 132001 (2007). arXiv:0704.0638 [hep-ex]

80. G.T. Bodwin, K.-T. Chao, H.S. Chung, U.-R. Kim, J. Lee, Y.-Q. Ma, Fragmentation contributions to hadroproduction of prompt $J / \psi$, $\chi_{c J}$, and $\psi(2 S)$ states. Phys. Rev. D 93(3), 034041 (2016). arXiv:1509.07904 [hep-ph]

81. N. Yamanaka, J.-P. Lansberg, H.-S. Shao, Y.-J. Zhang, Studying parton correlations via double parton scatterings in associated quarkonium production at the LHC and the Tevatron. In 39th International Conference on High Energy Physics (ICHEP 2018) Seoul, Korea, July 4-11, 2018 (2018). arXiv:1811.07474 [hep-ph]

82. J.-P. Lansberg, H.-S. Shao, N. Yamanaka, Y.-J. Zhang, $J / \psi+J / \psi$, $J / \psi+\Upsilon, \Upsilon+\Upsilon$ production at the LHC and Tevatron at one-loop in a quark-hadron-duality approach (in preparation) (2019) 